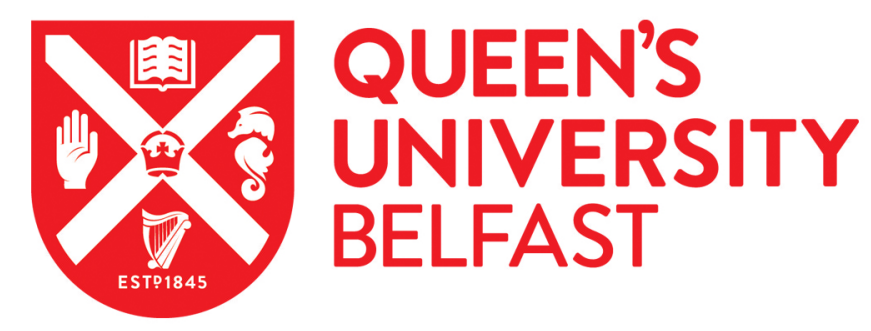

\title{
1-amenability of $A(X)$ for Banach spaces with 1-unconditional bases
}

Blanco, A. (2012). 1-amenability of $A(X)$ for Banach spaces with 1-unconditional bases. Studia Mathematica, 213(2), 97-131. https://doi.org/10.4064/sm213-2-1

Published in:

Studia Mathematica

Document Version:

Early version, also known as pre-print

Queen's University Belfast - Research Portal:

Link to publication record in Queen's University Belfast Research Portal

\section{General rights}

Copyright for the publications made accessible via the Queen's University Belfast Research Portal is retained by the author(s) and / or other copyright owners and it is a condition of accessing these publications that users recognise and abide by the legal requirements associated with these rights.

Take down policy

The Research Portal is Queen's institutional repository that provides access to Queen's research output. Every effort has been made to ensure that content in the Research Portal does not infringe any person's rights, or applicable UK laws. If you discover content in the Research Portal that you believe breaches copyright or violates any law, please contact openaccess@qub.ac.uk. 


\title{
1-amenability of $\mathcal{A}(X)$ for Banach spaces with 1-unconditional bases
}

by

\author{
A. Blanco (Belfast)
}

\begin{abstract}
The main result of the note is a characterization of 1-amenability of Banach algebras of approximable operators for a class of Banach spaces with 1-unconditional bases in terms of a new basis property. It is also shown that amenability and symmetric amenability are equivalent concepts for Banach algebras of approximable operators, and that a type of Banach space that was long suspected to lack property $\mathbb{A}$ has in fact the property. Some further ideas on the problem of whether or not amenability (in this setting) implies property $\mathbb{A}$ are discussed.
\end{abstract}

1. Introduction. The homological notion of an amenable Banach algebra was introduced by Johnson in [J1] and it has been the object of much study ever since. A Banach algebra $\mathcal{A}$ is said to be amenable if every continuous derivation from $\mathcal{A}$ into a dual Banach $\mathcal{A}$-bimodule is inner, or equivalently, if the first (continuous) cohomology group of $\mathcal{A}$ with coefficients in any dual $\mathcal{A}$-bimodule is trivial.

In the case of Banach algebras that sit on some particular structure there has been much interest in characterizing amenability in terms of properties of the underlying structure. The best example of this kind is the celebrated result of Johnson that the group algebra, $L^{1}(G)$, of a locally compact group $G$ is amenable if and only if the group $G$ is amenable [J1, Theorem 2.5]. One would expect an analogous characterization to be possible for the Banach algebras $\mathcal{A}(X)$ and $\mathcal{B}(X)$ of approximable and bounded operators, respectively, acting on a Banach space $X$, but to date, we do not know of any such result.

In this note, our main concern will be the Banach algebra $\mathcal{A}(X)$. The study of amenability of this algebra was initiated in [J1] and then continued in [GJW], where a certain Banach space property, which the authors called property $\mathbb{A}$, was identified and shown to be a sufficient condition for

2010 Mathematics Subject Classification: Primary 46B20, 46B28, 47L10; Secondary 16E40. Key words and phrases: amenability, approximable operator, Banach algebra, Banach space. 
amenability of $\mathcal{A}(X)$. Later on, in [BG2], we showed that a weaker version of property $\mathbb{A}$ was, in fact, equivalent to amenability of $\mathcal{A}(X)$ whenever $X$ is a $\pi$-space. This new condition was compared with the Følner condition for group amenability. However, still this characterization was not entirely satisfactory. It reduced the problem of amenability of $\mathcal{A}(X)$ to the existence of a bounded net of so-called generalized diagonals. These objects are elements of projective tensor products of the form $\mathcal{F}(Y, Z) \widehat{\otimes} \mathcal{F}(Z, Y)$, where $Y$ and $Z$ are finite-dimensional subspaces of $X$. Although they are well described from the algebraic point of view, the intractability of the projective norm (at least for practical purposes) led us to abandon any hope of being able to further translate our condition into a geometric property of the underlying Banach space.

In this paper, we shall be able to translate 1-amenability of $\mathcal{A}(X)$ for a wide class of Banach spaces with 1-unconditional bases into a property of the basis which is much in the spirit of property $\mathbb{A}$. It has long been known that local unconditional structure of the underlying space is not required for amenability of $\mathcal{A}(X)$ ([GJW, Corollary 2.7]). However, in its full generality, the problem mentioned at the end of the previous paragraph seems to be rather difficult. For this reason, we have chosen to start by looking at Banach spaces with 1-unconditional bases. It is known that there are Banach spaces with 1-unconditional bases whose algebras of approximable operators are not amenable ([BG2, Theorem 5.6]) while, on the other hand, if $X$ has a subsymmetric basis (or even less, if $X$ has a 1-unconditional basis such that the right and left shift operators with respect to it are power bounded) then $\mathcal{A}(X)$ is amenable. So even in this restrictive setting the answer to our problem is far from trivial.

We should point out that also the 1-amenability assumption will play a key role in our results. It is essentially this condition that will allow us to translate the existence of a sequence of generalized diagonals into a property of the given 1-unconditional basis. In doing so, we shall rely heavily on approximate versions of known results about norm-one projections on Banach spaces with 1-unconditional bases as well as on known results about the extreme properties of the identity element of a Banach algebra. We do not know if whenever $\mathcal{A}(X)$ is amenable, an equivalent renorming, $\widetilde{X}$, of $X$ exists such that $\mathcal{A}(\tilde{X})$ is 1-amenable and, in case of $X$ having a 1-unconditional basis, such that, in addition, the unconditionality constant be preserved. If the answer to this last question were negative (as we suspect) then it seems very unlikely that our results will still hold if one removes the 1-unconditionality and 1-amenability assumptions.

Lastly, the note has been organized as follows. In the next section, we will cover some of the background material and terminology needed throughout these pages. Section 3 might seem to the reader like a small digression, but 
we feel it belongs in here. In it, we will look at the relationship between amenability and symmetric amenability in the context of Banach algebras of approximable operators. The notion of symmetric amenability was introduced in J3 and was expected to be (in this context) stronger than amenability and closely related to property $\mathbb{A}$. We will show in Section 3 that this is not the case. We will then turn our attention, in Section 4, to the finite-dimensional situation of our main problem. It will be shown in this section that if $X$ is finite-dimensional and has a 1-unconditional basis then 1-amenability of $\mathcal{A}(X)$ is equivalent to $X$ having property $\mathbb{A}$ with constant 1 .

In Section 5, we will prove the main result of the note, i.e., our characterization of 1-amenability of $\mathcal{A}(X)$ for a Banach space $X$ with a 1unconditional basis in terms of a basis property. Unfortunately, apart from 1-unconditionality, other assumptions on the basis will be needed. We will introduce and discuss them also in Section 5. The last section of the paper, Section 6 , will be devoted to the question of whether property $\mathbb{A}$ is a necessary condition for amenability of $\mathcal{A}(X)$. This question goes back to [GJW] and is closely related to the main problem of this note. Although we have been unable to answer it, we expect the results of this paper, and in particular those from the last section, will help addressing this seemingly simpler question.

2. Background material and notation. The notation and terminology that we will use in what follows are standard and mostly consistent with those used in previous papers. Let us recall here some of them. Others will be introduced or recalled later on as they are needed.

To simplify the statement of the results, we shall denote by $\ell_{\infty}$ the linear space, usually denoted by $c_{0}$, of all bounded scalar sequences tending to zero. Given a normed space $X$ we denote by $X^{\prime}$ its topological dual. If $X$ and $Y$ are isomorphic (resp., isometric) normed spaces, we write this as $X \simeq Y$ (resp., $X \cong Y$ ), and denote by $d(X, Y)$ the Banach-Mazur distance between them, that is, the infimum of the numbers $\|T\|\left\|T^{-1}\right\|$, where $T$ is an isomorphism between $X$ and $Y$.

The adjoint of an operator $T: X \rightarrow Y$ is denoted by $T^{*}$ and we write $\operatorname{rg} T$ (resp., $\operatorname{rk} T$ ) for the range (resp., rank) of $T$. The identity operator on a normed space $X$ will be denoted by $\operatorname{id}_{X}$ or just id if the space $X$ is clear from context. Given a linear map $T: X \rightarrow Y$ and linear subspaces $E \subseteq X$ and $F \subseteq Y$ we will denote by $\left.T\right|_{E}$ and $\left.T\right|^{F}$, respectively, the restriction to $E$ and corestriction to $F$ of $T$.

We write $\|\cdot\|_{\wedge}$ (resp., $\left.\|\cdot\|\right)$ for the projective (resp., operator) norm. Given a subset $S$ of a Banach space $X$, we write $\operatorname{sp}(S)$ for its linear span and $\overline{\mathrm{co}}(S)$ for the norm-closure of its convex hull. In the case of an indexed 
subset $\left\{e_{i}: i \in I\right\}$ of $X$, we write $\left[e_{i}\right]_{i \in I}$ (or $\left[e_{i}: i \in I\right]$ ) for the closure of its linear span. We denote by $X_{[1]}$ the (closed) unit ball of a normed space $X$. We write $\left(x_{i}\right)_{i=1}^{n}$ for the sequence consisting of the elements $x_{1}, \ldots, x_{n}$, or just $\left(x_{i}\right)$ if $n$ is clear from context.

Given Banach spaces $X$ and $Y$ we write $\mathcal{F}(X, Y)$ for the normed space of all finite-rank operators from $X$ to $Y$ endowed with the operator norm. When appropriate we will identify $\mathcal{F}(X, Y)$ with $X^{\prime} \otimes Y$, so that, for $f \in X^{\prime}$ and $y \in Y$, the rank-1 operator $x \mapsto f(x) y$ will be denoted by $f \otimes y$. When $X=Y$ we simply write $\mathcal{F}(X)$. We will denote by $\operatorname{tr}: X^{\prime} \widehat{\otimes} X \rightarrow \mathbb{C}$ the trace functional, i.e., the linear functional defined on elementary tensors by $\operatorname{tr}(f \otimes x):=f(x)$.

There is an intrinsic characterization of amenability that is particularly useful in this setting and will be crucial to the results of this paper. Precisely, a Banach algebra $\mathcal{A}$ is amenable if and only if it has a bounded approximate diagonal, i.e., a bounded net $\left(d_{\alpha}\right)$ in $\mathcal{A} \widehat{\otimes} \mathcal{A}$ such that

$$
\pi\left(d_{\alpha}\right) a \rightarrow a \text { and } a d_{\alpha}-d_{\alpha} a \rightarrow 0 \quad(a \in \mathcal{A}),
$$

where $\pi: \mathcal{A} \widehat{\otimes} \mathcal{A} \rightarrow \mathcal{A}, a \otimes b \mapsto a b$ [J2, Lemma 1.2 and Theorem 1.3]. The Banach algebra $\mathcal{A}$ is said to be $K$-amenable if it has an approximate diagonal of bound $K$. The smallest such $K$ is called the amenability constant of $\mathcal{A}$. Recall that a bounded approximate identity (b.a.i. for short) for a Banach algebra $\mathcal{A}$ is a bounded net $\left(e_{\alpha}\right)$ in $\mathcal{A}$ such that $\lim _{\alpha} e_{\alpha} a=\lim _{\alpha} a e_{\alpha}=a$ $(a \in \mathcal{A})$. So, in particular, if $\left(d_{\alpha}\right)$ is a bounded approximate diagonal for $\mathcal{A}$ then $\left(\pi\left(d_{\alpha}\right)\right)$ is a b.a.i. for $\mathcal{A}$.

Let $^{\circ}: \mathcal{A} \widehat{\otimes} \mathcal{A} \rightarrow \mathcal{A} \widehat{\otimes} \mathcal{A}$ be defined by $(a \otimes b)^{\circ}:=b \otimes a(a \otimes b \in \mathcal{A} \widehat{\otimes} \mathcal{A})$. The algebra $\mathcal{A}$ is said to be symmetrically amenable if it has a bounded approximate diagonal $\left(d_{\alpha}\right)$ all of whose elements satisfy $d_{\alpha}^{\circ}=d_{\alpha}$. An approximate diagonal with this last property is said to be symmetric. In general, symmetric amenability is stronger than amenability. For commutative Banach algebras and group algebras they agree ([J3, Corollary 2.3 and Theorem 4.1]) and we will see below that they also agree for Banach algebras of approximable operators.

A Banach space $X$ is said to have property $\mathbb{A}$ if there is in $\mathcal{A}(X)$ a bounded net $\left(p_{\alpha}\right)$ of projections such that:

(i) $\left(p_{\alpha}\right)$ converges strongly to $\mathrm{id}_{X}$;

(ii) $\left(p_{\alpha}^{*}\right)$ converges strongly to $\mathrm{id}_{X^{\prime}}$; and

(iii) for every $\alpha$ there is a finite group $G_{\alpha} \subset \mathcal{A}\left(\operatorname{rg} p_{\alpha}\right)_{[K]}$ such that $\operatorname{sp}\left(G_{\alpha}\right)=\mathcal{A}\left(\operatorname{rg} p_{\alpha}\right)$, where $K$ is a constant independent of $\alpha$.

It is easy to show that, in this case, the elements $\left|G_{\alpha}\right|^{-1} \sum_{T \in G_{\alpha}} T \otimes T^{-1}$ form a symmetric approximate diagonal for $\mathcal{A}(X)$ of bound $K$. We shall refer to the smallest constant $K$ for which (i)-(iii) hold as the property $\mathbb{A}$ constant 
of $X$. Recall also that a Banach space $X$ such that $\mathcal{A}(X)$ contains a bounded net of projections converging strongly to $\mathrm{id}_{X}$ is said to be a $\pi$-space.

Given a pair of finite-dimensional Banach spaces $X$ and $Y$, an element $d$ of $\mathcal{F}(Y, X) \widehat{\otimes} \mathcal{F}(X, Y)$ is called a generalized diagonal for $\mathcal{F}(X)$ if $\pi(d)=\mathrm{id}_{X}$ and $W d=d W(W \in \mathcal{F}(X))$. If $\left(x_{k}\right)_{k=1}^{m}$ and $\left(y_{i}\right)_{i=1}^{n}$ are bases for $X$ and $Y$, respectively, and $d$ is a generalized diagonal, then $d$ can be represented in the form $\sum_{i, j} a_{i, j} \sum_{k}\left(y_{j}^{*} \otimes x_{k}\right) \otimes\left(x_{k}^{*} \otimes y_{i}\right)$, where the $a_{i, j}$ 's are scalars such that $\sum_{i} a_{i, i}=1$. Conversely, any element of this form is a generalized diagonal for $\mathcal{F}(X)$. There is in $\mathcal{F}(X) \widehat{\otimes} \mathcal{F}(X)$ a unique generalized diagonal for $\mathcal{F}(X)$, $d$ say, with the additional property that $d^{\circ}$ is also a generalized diagonal for $\mathcal{F}(X)$. We shall refer to this unique element as the symmetric diagonal for $\mathcal{F}(X)$.

It was shown in [BG2, Corollary 3.3] that if $X$ is a Banach space with a bounded net $\left(p_{\alpha}\right)_{\alpha \in I}$ of projections converging strongly to $\mathrm{id}_{X}$ and such that $\left(p_{\alpha}^{*}\right)_{\alpha \in I}$ converges strongly to $\operatorname{id}_{X^{\prime}}$ then $\mathcal{A}(X)$ is amenable if and only if there is a constant $K \geq 1$ such that, for every $\alpha \in I$, there is $\beta \in I$ such that $\mathcal{F}\left(\operatorname{rg} p_{\beta}, \operatorname{rg} p_{\alpha}\right) \widehat{\otimes} \mathcal{F}\left(\operatorname{rg} p_{\alpha}, \operatorname{rg} p_{\beta}\right)$ contains a generalized diagonal $d_{\alpha}$ for $\mathcal{F}\left(\operatorname{rg} p_{\alpha}\right)$ of norm $\leq K$. Moreover, if $\mathcal{A}(X)$ is 1-amenable and $\lim _{\alpha}\left\|p_{\alpha}\right\|=1$ then, given an arbitrary net $\left(\varepsilon_{\alpha}\right)$ of positive real numbers converging to zero, the $d_{\alpha}$ 's can be chosen to satisfy $\left\|d_{\alpha}\right\|_{\wedge} \leq 1+\varepsilon_{\alpha}$. The latter was not explicitly stated in [BG2, but it is implicit in the proof of [BG2, Proposition 3.1].

Recall that a Schauder basis $\left(x_{i}\right)$ for a Banach space $X$ is said to be 1-unconditional if

$$
\left\|\sum_{i} \alpha_{i} x_{i}\right\| \leq\left\|\sum_{i} \beta_{i} x_{i}\right\|
$$

whenever $\left(\alpha_{i}\right)$ and $\left(\beta_{i}\right)$ are (eventually null) scalar sequences with $\left|\alpha_{i}\right| \leq\left|\beta_{i}\right|$ $(i \in \mathbb{N})$. If $\left\|x_{i}\right\|=1(i \in \mathbb{N})$ then the basis is said to be normalized. Given a basis $\left(x_{i}\right)$ we write $x_{k}^{*}$ for the $k$ th biorthogonal functional associated with it. If the associated sequence $\left(x_{i}^{*}\right)$ of biorthogonal functionals forms a basis for $X^{\prime}$ then we call the basis shrinking.

If $X$ is a Banach space with a Schauder basis $\left(x_{i}\right)$ then we denote by $\operatorname{supp} x$ the support of a vector $x \in X$, i.e., the set $\left\{i \in \mathbb{N}: x_{i}^{*}(x) \neq 0\right\}$. Vectors $x$ and $y$ will be said to be disjointly supported if $\operatorname{supp} x \cap \operatorname{supp} y=\emptyset$. By a support functional for a given vector $x \in X$ we always mean a normone linear functional $f \in X^{\prime}$ such that $f(x)=\|x\|$. As is customary while working with sequence spaces, if $E$ and $F$ are non-empty subsets of $\mathbb{N}$, we write $E<F$ to mean $\max E<\min F$ and denote also by $E$ the projection $\sum_{i} \alpha_{i} x_{i} \mapsto \sum_{i \in E} \alpha_{i} x_{i}$

Given a Banach space $X$ with a 1-unconditional Schauder basis $\left(x_{i}\right)$, there is, for each pair of positive integers $m<n$, a natural isometric Banach algebra embedding $\mathcal{A}\left(\left[x_{i}\right]_{i=1}^{m}\right) \hookrightarrow \mathcal{A}\left(\left[x_{i}\right]_{i=1}^{n}\right)$ induced by the natural 
embedding $\left[x_{i}\right]_{i=1}^{m} \hookrightarrow\left[x_{i}\right]_{i=1}^{n}$. Letting $m$ and $n$ vary we obtain a direct system of Banach algebras and isometric Banach algebra homomorphisms. Its inductive limit is also a Banach algebra that we shall denote by $\mathcal{A}_{0}(X)$. In general, $\mathcal{A}_{0}(X)$ is properly contained in $\mathcal{A}(X)$, but if $\left(x_{i}\right)$ is shrinking then $\mathcal{A}_{0}(X)=\mathcal{A}(X)$. When $\mathcal{A}(X)$ is selfinduced (see [BG1, Section 1.2] for the definition) the amenability of $\mathcal{A}(X)$ can be deduced from that of $\mathcal{A}_{0}(X)$ ([BG1, Lemma 2.2]).

Since we will rely on [BG2, Corollary 3.3] we will need the natural projections with respect to a given basis for $X$ to form a b.a.i. for $\mathcal{A}(X)$. However, rather than assuming this (or equivalently, that the basis is shrinking), it seems more natural to us to state our results for $\mathcal{A}_{0}(X)$, and this is precisely what we will do.

Lastly, in what follows, operator always means continuous linear operator and we assume all our Banach spaces to be over the complex field. We write $\mathbb{R}_{+}$for the set $\{t \in \mathbb{R}: t>0\}$.

3. Symmetric amenability of $\mathcal{A}(X)$. There is no obvious reason at all to expect that whenever a bounded approximate diagonal for $\mathcal{A}(X)$ exists there is also a symmetric one. However, as we will see next, this happens to be the case.

Theorem 3.1. Let $X$ be a Banach space. Then $\mathcal{A}(X)$ is amenable if and only if it is symmetrically amenable.

Proof. In what follows, mainly to simplify notation, we shall identify $\mathcal{F}(X)$ with $X^{\prime} \otimes X$ (see fifth paragraph of the previous section). It is with this identification in mind that we shall apply $\|\cdot\|_{\wedge}$ and tr to operators in $\mathcal{F}(X)$.

Of course, we only need to show that if $\mathcal{A}(X)$ is amenable then it is symmetrically amenable. To this end, let $\left(d_{\alpha}\right) \subset \mathcal{F}(X) \otimes \mathcal{F}(X)$ be a bounded approximate diagonal for $\mathcal{A}:=\mathcal{A}(X)$, so $\left(\pi\left(d_{\alpha}\right)\right)$ is a b.a.i. for $\mathcal{A}$. For each $\alpha$, set $e_{\alpha}=\pi\left(d_{\alpha}\right)$ and choose a projection $p_{\alpha} \in \mathcal{A}$ so that $p_{\alpha} e_{\alpha}=e_{\alpha}$ and

$$
\frac{\left\|e_{\alpha}\left(e_{\alpha}-2 \mathrm{id}\right)\right\|_{\wedge}\left\|p_{\alpha}\right\|}{\operatorname{tr} p_{\alpha}} \leq \varepsilon_{\alpha}:=1 / \mathrm{rk} e_{\alpha} .
$$

This is possible because for every finite-dimensional subspace $E$ of $X$ there exists a projection onto it of norm no greater than $\sqrt{\operatorname{dim} E}$ (see [Pi1, Theorem 1.14]). Also note that $\lim _{\alpha} \varepsilon_{\alpha}=0$. Choose $\gamma(=\gamma(\alpha))$ 'greater' than $\alpha$ such that

$$
\left|1-\frac{\operatorname{tr}\left(p_{\alpha} e_{\gamma}\right)}{\operatorname{tr} p_{\alpha}}\right| \leq \varepsilon_{\alpha},
$$

and such that, for some $\lambda_{\alpha} \in \mathbb{C}$, 


$$
\left\|\pi\left(\left(f_{\alpha} d_{\gamma}^{\circ} f_{\alpha}\right)^{\circ}\right) p_{\alpha}-\lambda_{\alpha} p_{\alpha}\right\|_{\wedge} \leq \varepsilon_{\alpha},
$$

where $f_{\alpha}=\mathrm{id}-e_{\alpha}$. That the latter is possible follows on noting that we have $w d_{\gamma}-d_{\gamma} w \rightarrow 0 \Rightarrow w \pi\left(\left(f_{\alpha} d_{\gamma}^{\circ} f_{\alpha}\right)^{\circ}\right)-\pi\left(\left(f_{\alpha} d_{\gamma}^{\circ} f_{\alpha}\right)^{\circ}\right) w \rightarrow 0$, for

$$
\begin{aligned}
\left\|w \pi\left(\left(f_{\alpha} d_{\gamma}^{\circ} f_{\alpha}\right)^{\circ}\right)-\pi\left(\left(f_{\alpha} d_{\gamma}^{\circ} f_{\alpha}\right)^{\circ}\right) w\right\| & =\left\|\pi\left(w\left(f_{\alpha} d_{\gamma}^{\circ} f_{\alpha}\right)^{\circ}-\left(f_{\alpha} d_{\gamma}^{\circ} f_{\alpha}\right)^{\circ} w\right)\right\| \\
& \leq\left\|w\left(f_{\alpha} d_{\gamma}^{\circ} f_{\alpha}\right)^{\circ}-\left(f_{\alpha} d_{\gamma}^{\circ} f_{\alpha}\right)^{\circ} w\right\|_{\wedge} \\
& \leq\left\|f_{\alpha}\right\|^{2}\left\|w d_{\gamma}-d_{\gamma} w\right\|_{\wedge} .
\end{aligned}
$$

Therefore, we can get $p_{\alpha} \pi\left(\left(f_{\alpha} d_{\gamma}^{\circ} f_{\alpha}\right)^{\circ}\right) p_{\alpha}$ as close to the center of $\mathcal{A}_{\alpha}:=$ $p_{\alpha} \mathcal{A} p_{\alpha}$ (i.e., as close to a multiple of $p_{\alpha}$ ) as we wish. Since

$p_{\alpha} \pi\left(\left(f_{\alpha} d_{\gamma}^{\circ} f_{\alpha}\right)^{\circ}\right) p_{\alpha}-\pi\left(\left(f_{\alpha} d_{\gamma}^{\circ} f_{\alpha}\right)^{\circ}\right) p_{\alpha}=\left(p_{\alpha} \pi\left(\left(f_{\alpha} d_{\gamma}^{\circ} f_{\alpha}\right)^{\circ}\right)-\pi\left(\left(f_{\alpha} d_{\gamma}^{\circ} f_{\alpha}\right)^{\circ}\right) p_{\alpha}\right) p_{\alpha}$ and $\mathcal{A}_{\alpha}$ is finite-dimensional, the same holds for $\pi\left(\left(f_{\alpha} d_{\gamma}^{\circ} f_{\alpha}\right)^{\circ}\right) p_{\alpha}$.

For each $\alpha$, define

$$
\delta_{\alpha}:=\left(f_{\alpha} d_{\gamma}^{\circ} f_{\alpha}\right)^{\circ}+f_{\alpha} d_{\gamma}^{\circ} f_{\alpha} .
$$

We show next that the net $\left(\delta_{\alpha}\right)$ is a (bounded) symmetric approximate diagonal for $\mathcal{A}$. That $\delta_{\alpha}^{\circ}=\delta_{\alpha}$ for every $\alpha$ is easy to check. To see that $w \delta_{\alpha}-\delta_{\alpha} w \underset{\alpha}{\rightarrow} 0(w \in \mathcal{A})$, simply note that

$$
\begin{aligned}
\left\|w \delta_{\alpha}-\delta_{\alpha} w\right\|_{\wedge} & \leq\left\|w\left(f_{\alpha} d_{\gamma}^{\circ} f_{\alpha}\right)^{\circ}-\left(f_{\alpha} d_{\gamma}^{\circ} f_{\alpha}\right)^{\circ} w\right\|_{\wedge}+\left\|w f_{\alpha} d_{\gamma}^{\circ} f_{\alpha}-f_{\alpha} d_{\gamma}^{\circ} f_{\alpha} w\right\|_{\wedge} \\
& \leq\left\|f_{\alpha}\right\|^{2}\left\|w d_{\gamma}-d_{\gamma} w\right\|_{\wedge}+\left\|f_{\alpha}\right\|\left\|d_{\gamma}\right\|_{\wedge}\left(\left\|w f_{\alpha}\right\|+\left\|f_{\alpha} w\right\|\right),
\end{aligned}
$$

and the last expression tends to zero with $\alpha$ for every $w \in \mathcal{A}$.

It remains to show that $\left(\pi\left(\delta_{\alpha}\right)\right)$ is a b.a.i. for $\mathcal{A}$. For this, first note that

$$
\begin{aligned}
\operatorname{tr} \pi\left(\left(f_{\alpha} d_{\gamma}^{\circ} f_{\alpha}\right)^{\circ} p_{\alpha}\right) & =\operatorname{tr} \pi\left(f_{\alpha}\left(p_{\alpha} d_{\gamma}\right)^{\circ} f_{\alpha}\right)=\operatorname{tr}\left(f_{\alpha}^{2} \pi\left(\left(p_{\alpha} d_{\gamma}\right)^{\circ}\right)\right) \\
& =\operatorname{tr}\left(p_{\alpha} e_{\gamma}\right)+\operatorname{tr}\left(e_{\alpha}\left(e_{\alpha}-2 \mathrm{id}\right) \pi\left(\left(p_{\alpha} d_{\gamma}\right)^{\circ}\right)\right),
\end{aligned}
$$

so we have

$$
\begin{aligned}
\left(1-\lambda_{\alpha}\right) \operatorname{tr} p_{\alpha}=[ & {\left[\operatorname{tr} p_{\alpha}-\operatorname{tr}\left(p_{\alpha} e_{\gamma}\right)\right]+\left[\operatorname{tr} \pi\left(\left(f_{\alpha} d_{\gamma}^{\circ} f_{\alpha}\right)^{\circ} p_{\alpha}\right)-\lambda_{\alpha} \operatorname{tr} p_{\alpha}\right] } \\
& -\operatorname{tr}\left(e_{\alpha}\left(e_{\alpha}-2 \operatorname{id}\right) \pi\left(\left(p_{\alpha} d_{\gamma}\right)^{\circ}\right)\right)
\end{aligned}
$$

and in turn,

$$
\left|1-\lambda_{\alpha}\right| \leq \varepsilon_{\alpha}\left(2+\left\|d_{\gamma}\right\|_{\wedge}\right) .
$$

For every $w \in \mathcal{A}$, let $w_{\alpha}:=e_{\alpha} w$. Then

$$
\begin{aligned}
\left\|\pi\left(\delta_{\alpha}\right) w_{\alpha}-w_{\alpha}\right\| & =\left\|\pi\left(\left(f_{\alpha} d_{\gamma}^{\circ} f_{\alpha}\right)^{\circ}\right) w_{\alpha}+f_{\alpha} \pi\left(d_{\gamma}^{\circ}\right) f_{\alpha} w_{\alpha}-w_{\alpha}\right\| \\
& \leq\left\|\pi\left(\left(f_{\alpha} d_{\gamma}^{\circ} f_{\alpha}\right)^{\circ}\right) w_{\alpha}-w_{\alpha}\right\|+\left\|f_{\alpha}\right\|\left\|d_{\gamma}\right\|_{\wedge}\left\|f_{\alpha} w_{\alpha}\right\|,
\end{aligned}
$$

and 


$$
\begin{aligned}
\| \pi\left(\left(f_{\alpha} d_{\gamma}^{\circ} f_{\alpha}\right)^{\circ}\right) & w_{\alpha}-w_{\alpha} \| \\
& \leq\left\|\pi\left(\left(f_{\alpha} d_{\gamma}^{\circ} f_{\alpha}\right)^{\circ}\right) p_{\alpha} w_{\alpha}-\lambda_{\alpha} p_{\alpha} w_{\alpha}\right\|+\left\|\lambda_{\alpha} w_{\alpha}-w_{\alpha}\right\| \\
& \leq\left\|\pi\left(\left(f_{\alpha} d_{\gamma}^{\circ} f_{\alpha}\right)^{\circ}\right) p_{\alpha}-\lambda_{\alpha} p_{\alpha}\right\|\left\|w_{\alpha}\right\|+\left|1-\lambda_{\alpha}\right|\left\|w_{\alpha}\right\| \\
& \leq \varepsilon_{\alpha}\left(3+\left\|d_{\gamma}\right\|_{\wedge}\right)\left\|w_{\alpha}\right\| .
\end{aligned}
$$

Combining (3.1) and 3.2 we readily see that $\left\|\pi\left(\delta_{\alpha}\right) w_{\alpha}-w_{\alpha}\right\| \underset{\alpha}{\rightarrow} 0$.

To finish the proof simply note that

$$
\begin{aligned}
\left\|\pi\left(\delta_{\alpha}\right) w-w\right\| & \leq\left\|\pi\left(\delta_{\alpha}\right) w-\pi\left(\delta_{\alpha}\right) w_{\alpha}\right\|+\left\|\pi\left(\delta_{\alpha}\right) w_{\alpha}-w_{\alpha}\right\|+\left\|w_{\alpha}-w\right\| \\
& \leq\left(1+2\left\|f_{\alpha}\right\|^{2}\left\|d_{\gamma}\right\|_{\wedge}\right)\left\|w_{\alpha}-w\right\|+\left\|\pi\left(\delta_{\alpha}\right) w_{\alpha}-w_{\alpha}\right\|,
\end{aligned}
$$

and the last expression converges to 0 with $\alpha$.

Thus, it will make no difference whatsoever, while working with Banach algebras of approximable operators, to assume that the algebra is symmetrically amenable instead of just amenable. However, this does not mean that, in the case of $\pi$-spaces, we shall be able to find an approximate diagonal whose elements are simultaneously generalized diagonals and symmetric tensors. Indeed, our proof of Theorem 3.1 suggests that, in general, one might need to give up the property of each $\pi\left(d_{\alpha}\right)$ being a projection in order to symmetrize the terms of the approximate diagonal. In what follows, the existence of an approximate diagonal whose terms are generalized diagonals will be a far more useful property to us, and so we shall not be concerned again with the symmetric aspect.

4. 1-Amenability (the finite case). The results of this section are particular cases of those to be discussed in the next section. However, the proofs in the finite-dimensional case are rather simple and therefore more transparent. We expect, by doing things in this way, to ease the reading of the next section, where fairly often, due to technicalities proper to the infinitedimensional setting, the arguments will be long and involved. In addition, it seems worth pointing out that the answer to the question of whether 1-amenability implies property $\mathbb{A}$ turns out to be 'almost' affirmative in the finite-dimensional case, as we shall see in Theorem 4.4 and Corollary 4.5 below.

Our first lemma is well known. Recall first that, given a convex subset $C$ of a vector space, a point $x \in C$ is said to be an extreme point of $C$ if whenever $x \in\left[x_{1}, x_{2}\right] \subset C$ either $x=x_{1}$ or $x=x_{2}$.

Lemma 4.1. Let $x$ be an extreme point of a convex subset $C$ of a $B a$ nach space. If $\left(x_{i}\right)$ and $\left(\lambda_{i}\right)$ are sequences (possibly finite) in $C$ and $\mathbb{R}_{+}$, respectively, such that $x=\sum_{i} \lambda_{i} x_{i}$ and $\sum_{i} \lambda_{i}=1$ then $x_{j}=x(j \in \mathbb{N})$. 
Clearly, in the finite-dimensional situation, the net $\left(p_{\alpha}\right)$ figuring in the definition of property $\mathbb{A}$ can be replaced by the identity map, and (i) and (ii) hold trivially. So only the third condition is of interest in this case and our next two lemmas deal essentially with it.

Lemma 4.2. Let $\mathcal{A}$ be a Banach algebra and let $e \in \mathcal{A}$ be a non-zero idempotent. If $\sum_{i} u_{i} \otimes v_{i} \in e \mathcal{A} \widehat{\otimes} \mathcal{A} e$ is such that $\sum_{i}\left\|u_{i}\right\|\left\|v_{i}\right\|=1$ and $\sum_{i} u_{i} v_{i}=e$ then $u_{i} v_{i}=\left\|u_{i}\right\|\left\|v_{i}\right\|$ e for every $i$.

Proof. First note that

$$
1=\sum_{i}\left\|u_{i}\right\|\left\|v_{i}\right\| \geq \sum_{i}\left\|u_{i} v_{i}\right\| \geq\|e\| \geq 1,
$$

so $\sum_{i}\left\|u_{i} v_{i}\right\|=1$ and $\left\|u_{i}\right\|\left\|v_{i}\right\|=\left\|u_{i} v_{i}\right\|$ for every $i$. Next note that $e$ is the identity of $e \mathcal{A} e$, so it must be an extreme point of its unit ball (see Section 3 of $[\mathrm{Lu}])$. Since $\sum_{i}\left\|u_{i} v_{i}\right\|\left(\left\|u_{i} v_{i}\right\|^{-1} u_{i} v_{i}\right)=e$, we must have, by the previous lemma, $u_{i} v_{i}=\left\|u_{i} v_{i}\right\| e=\left\|u_{i}\right\|\left\|v_{i}\right\| e$ for every $i$.

Lemma 4.3. Let $\mathcal{A}$ be a finite-dimensional Banach algebra, let $e \in \mathcal{A}$ be an idempotent and let $\sum_{i} u_{i} \otimes v_{i} \in e \mathcal{A} \widehat{\otimes} \mathcal{A} e$ be such that $\sum_{i} v_{i} u_{i}$ is invertible and $\sum_{i} a u_{i} \otimes v_{i}=\sum_{i} u_{i} \otimes v_{i} a(a \in e \mathcal{A} e)$. Then $\operatorname{sp}\left\{u_{i}\right\}=e \mathcal{A}$ and $\operatorname{sp}\left\{v_{i}\right\}=\mathcal{A} e$.

Proof. Let $\sum_{i} u_{i} \otimes v_{i}$ be as in the hypotheses. Without loss of generality, suppose $v_{1}, \ldots, v_{n}$ form a basis for $\operatorname{sp}\left\{v_{i}\right\}$, so $v_{i}=\alpha_{i, 1} v_{1}+\cdots+\alpha_{i, n} v_{n}$ for every $i>n$. Then $\sum_{i} u_{i} \otimes v_{i}=\sum_{i=1}^{n}\left(u_{i}+\sum_{k>n} \alpha_{k, i} u_{k}\right) \otimes v_{i}$. Let $\widetilde{u}_{i}:=$ $u_{i}+\sum_{k>n} \alpha_{k, i} u_{k}(1 \leq i \leq n)$, and let $\mathcal{J}=\operatorname{sp}\left\{\widetilde{u}_{i}\right\}$. Clearly, $\mathcal{J} \subseteq \operatorname{sp}\left\{u_{i}\right\} \subseteq e \mathcal{A}$. Next note that, for every $a \in e \mathcal{A} e$ and every $1 \leq i \leq n, a \widetilde{u}_{i} \in \mathcal{J}$. Indeed, suppose otherwise, that there are $1 \leq i_{0} \leq n$ and $a \in \mathcal{A}$ such that $a \widetilde{u}_{i_{0}} \notin \mathcal{J}$. Choose $\phi \in(e \mathcal{A})^{\prime}$ and $\psi \in(\mathcal{A} e)^{\prime}$ such that $\phi(\mathcal{J})=\{0\}, \phi\left(a \widetilde{u}_{i_{0}}\right)=1$ and $\psi\left(v_{i}\right)=\delta_{i i_{0}}(1 \leq i \leq n)$. Then, applying the linear functional $\phi \otimes \psi$ to both sides of the identity $\sum_{i} a \widetilde{u}_{i} \otimes v_{i}=\sum_{i} \widetilde{u}_{i} \otimes v_{i} a$, we reach the absurd $1=0$.

Let $u$ be the invertible element $\sum_{i} v_{i} u_{i}$. Then, for every $a \in \mathcal{A}$, we find, using the result from the previous paragraph, that

$$
e a=\left(e a u^{-1}\right) u=\sum_{i}\left(e a u^{-1}\right) v_{i} u_{i}=\sum_{i=1}^{n}\left(e a u^{-1}\right) v_{i} \widetilde{u}_{i} \in \mathcal{J},
$$

i.e., $e \mathcal{A} \subseteq \mathcal{J}$. It follows that $\operatorname{sp}\left\{u_{i}\right\}=e \mathcal{A}$, as claimed.

That $\operatorname{sp}\left\{v_{i}\right\}=\mathcal{A} e$ is proved analogously.

Notice that the assumption that $\sum_{i} v_{i} u_{i}$ is invertible cannot just be dropped from the hypotheses of the last lemma. For instance, if $X$ is a finitedimensional Banach space with basis $\left(x_{i}\right)_{i=1}^{n}$ then $\Delta:=\sum_{k=1}^{n}\left(x_{1}^{*} \otimes x_{k}\right) \otimes$ $\left(x_{k}^{*} \otimes x_{1}\right)$ is a diagonal for $\mathcal{A}(X)$, but neither $\operatorname{sp}\left\{x_{k}^{*} \otimes x_{1}: 1 \leq k \leq n\right\}=\mathcal{A}(X)$ nor $\operatorname{sp}\left\{x_{1}^{*} \otimes x_{k}: 1 \leq k \leq n\right\}=\mathcal{A}(X)$. 
Now the main result of the section reads as follows.

TheOREM 4.4. A finite-dimensional Banach algebra $\mathcal{A}$ is 1-amenable if and only if there is a group $G$ of invertible elements in $\mathcal{A}_{[1]}$ such that $\operatorname{sp}(G)=\mathcal{A}$.

Proof. Of course, as $\mathcal{A}$ is finite-dimensional, amenability and contractibility of $\mathcal{A}$ are the same thing, so we can talk of a diagonal instead of an approximate one.

It is well known that if there is a group $G$ of invertible elements in $\mathcal{A}_{[1]}$ whose linear span is the whole of $\mathcal{A}$ then $\mathcal{A}$ is 1-amenable. Simply replace $G$, if necessary, by its norm-closure $\bar{G}$ and take as a norm-1 diagonal the element $\mu(\bar{G})^{-1} \int_{\bar{G}} g \otimes g^{-1} d \mu(g)$, where $\mu$ denotes the Haar measure on the group $\bar{G}$ endowed with the topology induced by the norm.

In the opposite direction, if $\mathcal{A}$ is 1-amenable then there is a diagonal $\Delta$ for $\mathcal{A}$ of norm 1 . Furthermore, as $\mathcal{A}$ is finite-dimensional, $\Delta$ admits a finite representation $\sum_{i=1}^{n} u_{i} \otimes v_{i}$ such that $\left\|\sum_{i} u_{i} \otimes v_{i}\right\|_{\wedge}=\sum_{i}\left\|u_{i}\right\|\left\|v_{i}\right\|$ (see [TJ, Proposition 8.6]). Let $e$ be the identity of $\mathcal{A}$. Then, by Lemma 4.2 $u_{i} v_{i}=\left\|u_{i}\right\|\left\|v_{i}\right\| e(1 \leq i \leq n)$. It follows from this, combined with the finite-dimensionality of $\mathcal{A}$, that $\left\|v_{i}\right\|^{-1} v_{i}=\left(\left\|u_{i}\right\|^{-1} u_{i}\right)^{-1}(1 \leq i \leq n)$, so also $\sum_{i} v_{i} u_{i}=e$. The group $G=\left\langle\left\|u_{i}\right\|^{-1} u_{i}: 1 \leq i \leq n\right\rangle$ is clearly contained in $\mathcal{A}_{[1]}$ and, by Lemma 4.3 its linear span must be the whole of $\mathcal{A}$.

We do not know whether Theorem 4.4 remains true if one replaces in it 1 by a larger constant. We think this is very unlikely to happen but we do not have any examples. Clearly, the answer to this question would be positive whenever there is a renorming $\widetilde{\mathcal{A}}$ of $\mathcal{A}$ with amenability constant 1 .

Also, we are unable to conclude anything, in general, about the finiteness of the group $G$ appearing in Theorem 4.4. In certain cases, though, this is possible, as our next result shows. Recall first that a Hilbert (or Hilbertian) component of a Banach space $X$ with a 1-unconditional basis $\left(x_{i}\right)$ is simply any maximal (in the sense of inclusion) linear subspace of the form $\left[x_{i}: i \in J\right], J \subseteq \mathbb{N}$, which is isometrically isomorphic to a Hilbert space. (This definition of Hilbert component is not the usual one, but it is equivalent and it will suffice for our subsequent work. For the standard definition, which involves the concept of a Hermitian projection, we refer the reader to [KW, Sections 3-5].) Hilbert components of dimension greater than 1 are said to be non-trivial. It is known (see [KW, Theorem 5.3]) that the Hilbert components of a Banach space with a 1unconditional basis form a (topological) direct sum decomposition of the space.

Corollary 4.5. Let $X$ be a finite-dimensional Banach space with a normalized 1-unconditional basis $\left(x_{i}\right)$ and without non-trivial Hilbert com- 
ponents. Then $\mathcal{A}(X)$ has property $\mathbb{A}$ with constant 1 if and only if $\mathcal{A}(X)$ is 1-amenable.

Proof. In view of Theorem 4.4, all that is needed is to show that if $\mathcal{A}$ is 1 -amenable then $G$ can be chosen to be finite. Let $G=\left\langle\left\|u_{i}\right\|^{-1} u_{i}\right.$ : $1 \leq i \leq n\rangle$ be the group constructed in the proof of Theorem 4.4, so each $\left\|u_{i}\right\|^{-1} u_{i}$ is a linear isometry. In the special case under consideration, all linear isometries $T: X \rightarrow X$ are of the form $T\left(\sum_{i} \alpha_{i} x_{i}\right)=\sum_{i} \lambda_{i} \alpha_{i} x_{\pi(i)}$, where $\pi$ is a permutation of the index set and the $\lambda_{i}$ 's are scalars of modulus one (see [KW, Theorem 6.1]). Thus, each element $\left\|u_{i}\right\|^{-1} u_{i}$ can be written as the product of a permutation operator, which we shall denote by $\Pi_{i}$, and a diagonal linear isometry. Clearly, we must have $\left\|\Pi_{i}\right\|=1(1 \leq i \leq n)$. For every $\eta \in\{-1,1\}^{\operatorname{dim} X}$, let $U_{\eta}: X \rightarrow X$ be defined by $U_{\eta}\left(\sum_{i} \alpha_{i} x_{i}\right):=$ $\sum_{i} \eta_{i} \alpha_{i} x_{i}$. It is easy to see that $G_{0}:=\left\langle U_{\eta} \Pi_{i}: 1 \leq i \leq n, \eta \in\{-1,1\}^{\operatorname{dim} X}\right\rangle$ is finite, that $G_{0} \subset \mathcal{A}(X)_{[1]}$ and that $\operatorname{sp} G_{0}=\mathcal{A}(X)$ (the latter follows on noting that, as $\operatorname{sp} G=\mathcal{A}(X)$, there is, for every pair $1 \leq i, j \leq \operatorname{dim} X$, an element $\left\|u_{k}\right\|^{-1} u_{k} \in G$ such that $x_{i}^{*}\left(u_{k} x_{j}\right) \neq 0$, and therefore the same must hold with respect to $G_{0}$ ). The rest is clear.

The absence of non-trivial Hilbertian components is not a major requirement in Corollary 4.5, and can be dropped without affecting the rest of it. Indeed, by [ST, 2.3], if $X_{1}, \ldots, X_{m}$ are the Hilbert components of $X$ then there is a normed space $Y$ with a 1-unconditional basis $\left(y_{i}\right)_{i=1}^{m}$ such that $\left\|\left(x_{i}\right)_{i=1}^{m}\right\|_{X}=\left\|\sum_{i=1}^{m}\right\| x_{i}\left\|y_{i}\right\|_{Y}\left(\left(x_{i}\right) \in \bigoplus_{i=1}^{m} X_{i}\right)$ and every isometry $T: X \rightarrow X$ is of the form $T\left(\left(x_{i}\right)_{i=1}^{m}\right)=\left(U_{i} x_{\pi(i)}\right)_{i=1}^{m}$, where $\pi$ is a permutation and $U_{1}, \ldots, U_{m}$ are unitary operators. If $\mathcal{A}(X)$ is 1 -amenable then, combining these facts with the fact that the group $G$ of the proof of Corollary 4.5 must span the whole of $\mathcal{A}(X)$, one finds that all Hilbert components must be of the same dimension, and also that, if necessary, the group $G$ can be replaced by a finite one that still spans $\mathcal{A}(X)$. (For this last, one simply needs to note that every operator $T: X \rightarrow X$ of the form $T\left(\left(x_{i}\right)_{i=1}^{m}\right):=\left(U_{i} x_{i}\right)_{i=1}^{m}$, where $U_{1}, \ldots, U_{m}$ are unitaries, is an isometry.) Unfortunately, it is not the same in the infinite-dimensional case, where instead one needs to deal with non-surjective (almost) isometric maps. For this reason, in this note, we shall restrict our attention to Banach spaces all of whose Hilbert components are trivial.

\section{1-Amenability of $\mathcal{A}_{0}(X)$ when $X$ has a 1-unconditional basis.} We now extend the results from the previous section to the infinite-dimensional setting. From the characterization of amenability of $\mathcal{A}(X)$ for $\pi$-spaces, highlighted in Section 2, it follows easily that if $X$ is a Banach space with a 1-unconditional basis $\left(x_{i}\right)$ and $X_{n}:=\left[x_{i}\right]_{i=1}^{n}(n \in \mathbb{N})$ then $\mathcal{A}_{0}(X)$ is 1amenable if and only if, for every $n \in \mathbb{N}$, there exist $m \in \mathbb{N}$ and a generalized 
diagonal $d_{n} \in \mathcal{F}\left(X_{m}, X_{n}\right) \widehat{\otimes} \mathcal{F}\left(X_{n}, X_{m}\right)$ such that $\lim _{n}\left\|d_{n}\right\|_{\wedge}=1$. Clearly, Lemmas 4.3 and 4.2 concern the more general situation of a generalized diagonal, but Lemma 4.3, which is central to the results from the previous section, assumes, in addition, that the norm of the generalized diagonal is one. We do not know whether the $d_{n}$ 's can always be chosen to have norm one, and consequently we need to modify the results from the previous section to take into account this new difficulty. It is apparent, though, from the proof of [BG2, Proposition 3.1] that, although finding $d_{n}$ 's of norm one might not always be possible, it is always possible (by choosing $m$ large enough) to find $d_{n}$ 's with norms as close to 1 as we wish, and we shall take advantage of this fact.

Our first two lemmas are analogs of Lemmas 4.1 and 4.2 , respectively. Recall that, given a closed convex subset $C$ of a Banach space $X$, a point $x \in C$ is said to be an exposed point of $C$ if there is a real-linear, real-valued, norm-one functional $f$ on $X$ such that $f(x)>f(c)(c \in C \backslash\{x\})$.

Lemma 5.1. Let $C$ be a compact convex subset of a Banach space $X$ and suppose $x$ is an exposed point of $C$. For every $\tau>0$ there exists a constant $\varkappa_{\tau}$ such that whenever $\left(x_{i}\right) \subset C$ and $\left(\lambda_{i}\right) \subset \mathbb{R}_{+}$are sequences (possibly finite) satisfying $x=\sum_{i} \lambda_{i} x_{i}$ and $\sum_{i} \lambda_{i} \geq 1$ one has

$$
\sum_{i:\left\|x-x_{i}\right\|>\tau} \lambda_{i} \leq \varkappa_{\tau}\left(\sum_{i} \lambda_{i}-1\right) .
$$

Proof. Let $\tau>0$ arbitrary and let $x,\left(x_{i}\right)$ and $\left(\lambda_{i}\right)$ be as in the hypotheses. Let $u_{i}=x-x_{i}(i \in \mathbb{N})$ and let $I=\left\{i:\left\|u_{i}\right\|>\tau\right\}$. As $x$ is an exposed point of $C$, there is a real-linear, real-valued, norm-one functional $f$ on $X$ such that $f(x)>f(c)(c \in C \backslash\{x\})$. Compactness of $C$ guarantees that $f(x)>\sup _{c \in C_{\tau}} f(c)$, where $C_{\tau}:=\overline{C \backslash\left(x+\tau X_{[1]}\right)}$. So,

$$
\begin{aligned}
\left(\sum_{i} \lambda_{i}-1\right)\|x\| & =\left\|\sum_{i} \lambda_{i} x-\sum_{i} \lambda_{i} x_{i}\right\|=\left\|\sum_{i} \lambda_{i} u_{i}\right\| \\
& \geq f\left(\sum_{i} \lambda_{i} u_{i}\right) \geq \sum_{i \in I} \lambda_{i} f\left(u_{i}\right) \\
& \geq\left(f(x)-\sup _{c \in C_{\tau}} f(c)\right) \sum_{i \in I} \lambda_{i} .
\end{aligned}
$$

We can take $\varkappa_{\tau}=\|x\| /\left(f(x)-\sup _{c \in C_{\tau}} f(c)\right)$.

It is not hard to see that an exposed point is always an extreme point but, unfortunately, the converse is not true, and the conclusion of Lemma 5.1 does not seem to hold under the weaker assumption of $x$ being an extreme point. Fortunately, for any finite-dimensional unital Banach algebra the identity element is an exposed point of its unit ball. (This seems to be known but we 
have not found any reference. For completeness, a proof is given as part of the proof of our next lemma.)

Lemma 5.2. Let $\mathcal{A}$ be a Banach algebra and let $e \in \mathcal{A}$ be a non-zero

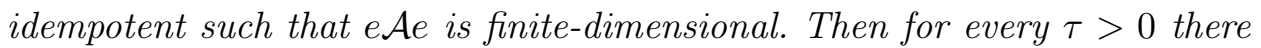
exists a constant $\varkappa_{\tau}$ such that whenever $\sum_{i} u_{i} \otimes v_{i} \in e \mathcal{A} \widehat{\otimes} \mathcal{A} e$ satisfies $\sum_{i} u_{i} v_{i}=e$ one has

$$
\sum_{i \in I_{\tau}}\left\|u_{i}\right\|\left\|v_{i}\right\| \leq \varkappa_{\tau}\left(\sum_{i}\left\|u_{i}\right\|\left\|v_{i}\right\|-1\right)
$$

where $I_{\tau}=\left\{i:\left\|\left(\left\|u_{i}\right\|^{-1} u_{i}\right)\left(\left\|v_{i}\right\|^{-1} v_{i}\right)-e\right\|>\tau\right\}$.

Proof. Let $C$ be the unit ball of $e \mathcal{A} e=: \mathfrak{A}$. Let us start by showing that $e$ is an exposed point of $C$. It is well known ([BK, Theorem 2]) that the set of all states of $\mathfrak{A}$ is total. Choose a basis of states $f_{1}, \ldots, f_{n}$ for $\mathfrak{A}^{\prime}$, choose $\lambda_{1}, \ldots, \lambda_{n} \in \mathbb{R}_{+}$such that $\sum_{i} \lambda_{i}=1$, and define $f:=\sum_{i} \lambda_{i} f_{i}$. Then

$$
\operatorname{Re} f(e)=1>\operatorname{Re} f(a) \quad(a \in C \backslash\{e\}) .
$$

To see this, suppose $\operatorname{Re} f(a)=1$ for some $a \in C$. Then $f(a)=1$, so $0=$ $f(e-a)=\sum_{i} \lambda_{i} f_{i}(e-a)$. Since $\operatorname{Re} f_{i}(e-a) \geq 0(1 \leq i \leq n)$, it follows from the last equality that $\operatorname{Re} f_{i}(e-a)=0(1 \leq i \leq n)$. In turn, using the fact that $f_{i}(e)=1(1 \leq i \leq n)$, we deduce that $f_{i}(e-a)=0(1 \leq i \leq n)$, and as the $f_{i}$ 's form a basis for $\mathfrak{A}^{\prime}$, we must have $e=a$. (The same argument can be easily adapted to any unital Banach algebra whose set of states contains a countable, weak ${ }^{*}$-dense subset. One simply replaces the basis of states in the above argument by this countable subset. The rest of the argument, apart from the obvious modifications, remains the same.)

Now, let $x=e$ and, for every $\tau>0$, define $\varkappa_{\tau}$ as in the previous lemma. If $\sum_{i} u_{i} \otimes v_{i}$ is such that $\sum_{i} u_{i} v_{i}=e$ then

$$
\sum_{i}\left\|u_{i}\right\|\left\|v_{i}\right\| \geq \sum_{i}\left\|u_{i} v_{i}\right\| \geq\|e\| \geq 1
$$

and

$$
e=\sum_{i}\left\|u_{i}\right\|\left\|v_{i}\right\|\left(\left\|u_{i}\right\|^{-1} u_{i}\right)\left(\left\|v_{i}\right\|^{-1} v_{i}\right),
$$

so $\sum_{i \in I_{\tau}}\left\|u_{i}\right\|\left\|v_{i}\right\| \leq \varkappa_{\tau}\left(\sum_{i}\left\|u_{i}\right\|\left\|v_{i}\right\|-1\right)$.

Notice that the finite-dimensionality assumption of Lemma 5.2 is important. For instance, in $\mathcal{B}\left(\ell_{2}\right)$, the identity is an exposed point of the unit ball [Gr, Theorem 1], but it is not denting [GS, Theorem 1] (a point $x$ of a subset $C$ of a Banach space $X$ is denting if, for every $\left.\delta>0, x \notin \overline{\mathrm{co}}\left(C \backslash\left\{x+\delta X_{[1]}\right\}\right)\right)$, and therefore a functional as in the proof of Lemma 5.1 cannot exist in this case.

As mentioned in the introduction, apart from 1-unconditionality, other assumptions on the basis will be needed for our approach to work. We dis- 
cuss next the most significant of these restrictions. Recall first that a 1unconditional basis $\left(x_{i}\right)$ for a Banach space $X$ is said to be strictly monotone if $\|x+y\|>\|x\|$ for every pair $x, y \in X_{+}$, where $X_{+}:=\left\{\sum_{i} \alpha_{i} x_{i} \in X\right.$ : $\left.\alpha_{i} \geq 0(i \in \mathbb{N})\right\}$.

Now, let $X$ and $Y$ be finite-dimensional Banach spaces with 1-unconditional bases $\left(x_{i}\right)$ and $\left(y_{j}\right)$, respectively, and let $u: Y \rightarrow X$ and $v: X \rightarrow Y$ be linear maps such that $u v=\operatorname{id}_{X}$ and $\|u\|\|v\|=1$. Then $\left.v\right|^{\operatorname{rg} v}$ is a surjective isometry and $v u$ is a norm-one projection onto $\operatorname{rg} v$. Suppose $X$ has no nontrivial Hilbert components and the basis $\left(y_{j}\right)$ is strictly monotone. First note that since $X$ has no non-trivial Hilbert components, the same must hold for $\operatorname{rg} v$, and hence, as the sequence $\left(v x_{i}\right)$ is a 1-unconditional basis for $\operatorname{rg} v$, each subspace $\left\langle v x_{i}\right\rangle$ must be a Hilbert component of $\operatorname{rg} v$. It is easy to see that $\operatorname{rg} v=\operatorname{rg} v u$, and so, by [Ra1, Theorem 3.1], the $v x_{i}$ 's must be disjointly supported with respect to $\left(y_{j}\right)$. It was precisely a particular case of this result that allowed us to translate the existence of a norm-1 diagonal into property $\mathbb{A}$, in the proof of Corollary 4.5. Now, it is apparent from Lemma 5.2 that, in the present situation, we will not be dealing necessarily with isometries, and so we will also need an approximate version of this result. To this end, we first introduce the following 'uniform' version of the concept of a strictly monotone basis.

Definition 5.3. Let $X$ be a Banach space with a normalized 1-unconditional basis $\left(x_{i}\right)$, and let $\mu_{X}:[0,1] \rightarrow[0,1]$ be defined by

$$
\mu_{X}(t):=\inf \left\{\|x+t y\|-1: x, y \in X_{+} \text {and }\|x\|=\|y\|=1\right\} .
$$

(It is easy to see that $\mu_{X}$ is non-decreasing and that $\lim _{t \rightarrow 0} \mu_{X}(t)=0$.) We shall say that the basis $\left(x_{i}\right)$ is uniformly strictly monotone (u.s.m. for short) if $\mu_{X}(t)>0(1 \geq t>0)$.

Any normalized 1-unconditional basis in a uniformly convex Banach space $X$, i.e., such that $\delta_{X}(t):=\inf \{1-\|(x+y) / 2\|:\|x\|=\|y\|=1$ and $\|x-y\| \geq t\}>0(2 \geq t>0)$, is uniformly strictly monotone. To see this, let $X$ be uniformly convex and suppose towards a contradiction that $\mu_{X}(t)=0$ for some $1 \geq t>0$. For every $n \in \mathbb{N}$, choose $x_{n}, y_{n} \in$ $X_{+}$such that $\left\|x_{n}\right\|=\left\|y_{n}\right\|=1$ and $\left\|x_{n}+t y_{n}\right\|-1 \leq 1 / n$, and set $\xi_{n}=\left(x_{n}+t y_{n}\right) /\left\|x_{n}+t y_{n}\right\|$. Then, for $n$ large enough, we should have $\left\|\xi_{n}-x_{n}\right\| \geq t / 2$, and hence $\left\|\left(x_{n}+\xi_{n}\right) / 2\right\| \leq 1-\delta_{X}(t / 2)$, while on the other hand, liminf $\left\|\left(x_{n}+\xi_{n}\right) / 2\right\|=\liminf \left\|x_{n}+(t / 2) y_{n}\right\| \geq 1$. Notice, though, that uniformly convex Banach spaces are not the only ones which can possibly have uniformly strictly monotone Schauder bases. For instance, $\mu_{\ell_{1}}(t)=t$ $(1 \geq t>0)$ and $\ell_{1}$ is not uniformly convex.

Before stating and proving our approximate version of the result discussed prior to Definition 5.3, we should recall a few things. First, the $n u$ - 
merical range of an operator $T$ acting on a Banach space $X$ is defined to be the set

$$
\mathcal{V}(T):=\left\{f(T x): x \in X, f \in X^{\prime},\|x\|=\|f\|=f(x)=1\right\}
$$

and its numerical radius is defined to be the number

$$
|\mathcal{V}(T)|:=\sup \{|z|: z \in \mathcal{V}(T)\} .
$$

When $X$ is a complex Banach space one has $\|T\| \leq 4|\mathcal{V}(T)|$ for every $T \in \mathcal{B}(X)([\mathrm{Lu}$, Theorem 5]). Recall also that a linear operator $T$ such that $\mathcal{V}(T) \subset \mathbb{R}$ is said to be Hermitian. In a Banach space with a 1-unconditional basis and without non-trivial Hilbertian components, the Hermitian operators are precisely those whose representation with respect to the given 1-unconditional basis is diagonal (see [KW, Theorem 6.1]).

Lemma 5.4. Let $X$ and $Y$ be finite-dimensional Banach spaces with normalized 1-unconditional bases $\left(x_{i}\right)_{i=1}^{m}$ and $\left(y_{i}\right)_{i=1}^{n}$, respectively. Suppose $X$ has no non-trivial Hilbertian components and $\left(y_{i}\right)$ is u.s.m. Then for every $\varepsilon>0$ there exists $\delta>0$, independent of $n$, such that if $V: Y \rightarrow X$ and $W: X \rightarrow Y$ are norm-one operators satisfying $\left\|V W-\operatorname{id}_{X}\right\| \leq \delta$ then there are pairwise disjoint subsets $E_{1}, \ldots, E_{m}$ of $\{1, \ldots, n\}$ such that

$$
\left\|W x_{i}-E_{i} W x_{i}\right\| \leq \varepsilon \quad(1 \leq i \leq m) .
$$

Proof. First note that, since $X$ has no non-trivial Hilbert components, $x_{i}^{*}\left(T x_{j}\right)=0(1 \leq i \neq j \leq m)$ for every Hermitian operator $T \in \mathcal{B}(X)$. Combining this fact with the finite-dimensionality of $\mathcal{B}(X)$, one easily deduced that, for every $\eta>0$ there is a $\bar{\delta}$ such that if $T \in \mathcal{B}(X)$ satisfies

$$
\mathcal{V}(T) \subset[-1,1]+\bar{\delta} \mathbb{C}_{[1]} \quad(=: G(\bar{\delta}))
$$

then $\left|x_{i}^{*}\left(T x_{j}\right)\right|<\eta(1 \leq i \neq j \leq m)$. Indeed, otherwise, for some $\eta>0$, there would be a sequence $\left(T_{n}\right)$ in $\mathcal{B}(X)$ such that $\mathcal{V}\left(T_{n}\right) \subset G(1 / n)$ yet $\max \left\{\left|x_{i}^{*}\left(T_{n} x_{j}\right)\right|: 1 \leq i \neq j \leq m\right\} \geq \eta(n \in \mathbb{N})$. Since $\mathcal{B}(X)$ is finitedimensional and $\left(T_{n}\right)$ is bounded, one could assume, by passing to a subsequence if necessary, that $\left(T_{n}\right)$ converges to some $T \in \mathcal{B}(X)$. Clearly, $T$ would be a Hermitian operator, and therefore $\lim _{n} x_{i}^{*}\left(T_{n} x_{j}\right)=x_{i}^{*}\left(T x_{j}\right)=0$ $(1 \leq i \neq j \leq m)$, which is a contradiction.

Now, let $\varepsilon>0$ be arbitrary. Choose $\bar{\varepsilon}>0$ small enough, so that $\mu_{Y}(t) \leq$ $8 \bar{\varepsilon} \Rightarrow t \leq \varepsilon /(2 m)$; choose $0<\bar{\delta}<9 \sqrt{\bar{\varepsilon}}$ so that for every $T \in \mathcal{B}(X)$ satisfying $\mathcal{V}(T) \subset G(\bar{\delta})$ one has $\left|x_{i}^{*}\left(T x_{j}\right)\right|<\bar{\varepsilon} /(m+1)(1 \leq i \neq j \leq m)$; and choose $0<\delta<\min \left\{1 / 2,(\bar{\delta} / 9)^{2}\right\}$. Let $V: Y \rightarrow X$ and $W: X \rightarrow Y$ be normone operators such that $\left\|V W-\operatorname{id}_{X}\right\| \leq \delta$, let $\xi_{i}=W x_{i}$ and $\xi_{i}^{*}=x_{i}^{*} V$ $(1 \leq i \leq m)$, and let $P=W V=\sum_{i=1}^{m} \xi_{i}^{*} \otimes \xi_{i}$. 
Let $(x, f) \in X \times X^{\prime}$ be such that $\|x\|=\|f\|=f(x)=1$. Then

$$
\left|1-\|f V\|^{-1} f V(W x)\right| \leq \frac{|\|f V\|-1|}{\|f V\|}+\frac{|f(x)-f V(W x)|}{\|f V\|} \leq \frac{2 \delta}{1-\delta} \leq 4 \delta,
$$

and, by the Bishop-Phelps-Bollobás theorem ([BD, Section 16, Theorem 1]) applied to the pair $\left(W x,\|f V\|^{-1} f V\right) \in Y \times Y^{\prime}$, there is $(y, g) \in Y \times Y^{\prime}$ such that $\|y\|=\|g\|=g(y)=1,\|W x-y\| \leq 4 \sqrt{\delta}$ and \|\|$f V\left\|^{-1} f V-g\right\| \leq 4 \sqrt{\delta}$. Note the last implies that

$$
\|f V-g\| \leq\|f V-\| f V\left\|^{-1} f V\right\|+\|\| f V\left\|^{-1} f V-g\right\| \leq \delta+4 \sqrt{\delta} \leq 5 \sqrt{\delta} .
$$

If $D$ is a norm-one operator on $Y$ then

$$
\begin{aligned}
|f(V D W x)-g(D y)| & \leq|f(V D W x)-g(D W x)|+|g(D W x)-g(D y)| \\
& \leq\|f V-g\|+\|W x-y\| \leq 9 \sqrt{\delta},
\end{aligned}
$$

and so if $D$ is in addition Hermitian, then $\mathcal{V}(V D W) \subset G(9 \sqrt{\delta})$. Thus, for every norm-one Hermitian $D \in \mathcal{B}(Y), \mathcal{V}(V D W) \subset G(\bar{\delta})$, and in turn, by our choice of $\bar{\delta}$,

$$
\left|\xi_{i}^{*}\left(D \xi_{j}\right)\right|=\left|x_{i}^{*}\left(V D W x_{j}\right)\right|<\frac{\bar{\varepsilon}}{m+1} \quad(1 \leq i \neq j \leq m) .
$$

Now, let $1 \leq k \neq l \leq m, \xi_{k}=\sum_{i} \alpha_{i} y_{i}$ and $\xi_{l}=\sum_{i} \beta_{i} y_{i}$. Define $\xi:=$ $\sum_{i} \gamma_{i} y_{i}$, where $\gamma_{i}:=\alpha_{i}\left|\beta_{i}\right| /\left(2\left|\alpha_{i}\right|\right)$ if $\left|\alpha_{i}\right|>\left|\beta_{i}\right|$ and $\gamma_{i}:=\alpha_{i} / 2$ otherwise. Note that for $E=\left\{1 \leq i \leq n:\left|\alpha_{i}\right|>\left|\beta_{i}\right|\right\}$ one has

$$
\left\|\xi_{k}-E \xi_{k}\right\| \leq 2\|\xi\| \quad \text { and } \quad\left\|E \xi_{l}\right\| \leq 2\|\xi\| \text {. }
$$

Let $D_{k}, D_{l 1}$ and $D_{l 2}$ be Hermitian maps in $\mathcal{B}(Y)$ of norm $\leq 1$ and such that $D_{k} \xi_{k}=\xi=\left(D_{l 1}+i D_{l 2}\right) \xi_{l}$. Then

$$
\begin{aligned}
\|P \xi\| & =\left\|\sum_{i=1}^{m} \xi_{i}^{*}(\xi) \xi_{i}\right\| \\
& \leq\left\|\sum_{i \neq k} \xi_{i}^{*}\left(D_{k} \xi_{k}\right) \xi_{i}+\left(\xi_{k}^{*}\left(D_{l 1} \xi_{l}\right)+i \xi_{k}^{*}\left(D_{l 2} \xi_{l}\right)\right) \xi_{k}\right\| \\
& \leq \sum_{i \neq k}\left|\xi_{i}^{*}\left(D_{k} \xi_{k}\right)\right|\left\|\xi_{i}\right\|+\left(\left|\xi_{k}^{*}\left(D_{l 1} \xi_{l}\right)\right|+\left|\xi_{k}^{*}\left(D_{l 2} \xi_{l}\right)\right|\right)\left\|\xi_{k}\right\| \leq \bar{\varepsilon} .
\end{aligned}
$$

It follows from this and the definition of $\mu_{Y}$ that

$$
\begin{aligned}
\left(1+\mu_{Y}\left(\frac{\|\xi\|}{\left\|\xi_{k}-\xi\right\|}\right)\right)\left\|\xi_{k}-\xi\right\| & \leq\left\|\xi_{k}\right\| \leq\left\|P \xi_{k}\right\|+\delta \\
& <\left\|P\left(\xi_{k}-\xi\right)+P \xi\right\|+\bar{\varepsilon} \leq\left\|\xi_{k}-\xi\right\|+2 \bar{\varepsilon}
\end{aligned}
$$

Thus,

$$
\mu_{Y}\left(\frac{\|\xi\|}{\left\|\xi_{k}-\xi\right\|}\right) \leq \frac{2 \bar{\varepsilon}}{\left\|\xi_{k}-\xi\right\|} \leq 8 \bar{\varepsilon}
$$


where we have used that $2\left\|\xi_{k}-\xi\right\| \geq\left\|\xi_{k}\right\| \geq 1-\delta \geq 1 / 2$. By our choice of $\bar{\varepsilon}$,

$$
\|\xi\| \leq \frac{\varepsilon}{2 m}\left\|\xi_{k}-\xi\right\| \leq \frac{\varepsilon}{2 m}\left\|\xi_{k}\right\| \leq \frac{\varepsilon}{2 m},
$$

and combining this last with $(5.1)$, we arrive at

$$
\left\|\xi_{k}-E \xi_{k}\right\| \leq \frac{\varepsilon}{m} \quad \text { and } \quad\left\|E \xi_{l}\right\| \leq \frac{\varepsilon}{m} .
$$

Now, for each pair $1 \leq i<j \leq m$, choose a subset $E_{i, j}$ of $\{1, \ldots, n\}$ with

$$
\left\|\xi_{i}-E_{i, j} \xi_{i}\right\| \leq \frac{\varepsilon}{m} \quad \text { and } \quad\left\|E_{i, j} \xi_{j}\right\| \leq \frac{\varepsilon}{m}
$$

(which is possible, by our previous result). Define $E_{i, j}:=E_{j, i}^{c}$ for $i>j$, and for $1 \leq i \leq m$, define $E_{i}:=\bigcap_{j \neq i} E_{i, j}$. Then

$$
\left\|\xi_{i}-E_{i} \xi_{i}\right\|=\left\|E_{i}^{c} \xi_{i}\right\|=\left\|\left(\bigcup_{j} E_{i, j}^{c}\right) \xi_{i}\right\| \leq \sum_{j}\left\|E_{i, j}^{c} \xi_{i}\right\|<\varepsilon \quad(1 \leq i \leq m),
$$

where the superscript ' $c$ ' stands for complement. Moreover,

$$
E_{k} \cap E_{l}=\left(\bigcap_{j \neq k} E_{k, j}\right) \cap\left(\bigcap_{j \neq l} E_{l, j}\right) \subset E_{k, l} \cap E_{l, k}=\emptyset \quad(1 \leq k \neq l \leq m),
$$

so, in addition, the sets $E_{1}, \ldots, E_{m}$ are pairwise disjoint, as needed. This concludes the proof of the lemma.

Recall that a biorthogonal system $\left\{x_{i}, f_{i}\right\}_{i=1}^{n}$ for a Banach space $X$ is a pair of sequences $\left(x_{i}\right)_{i=1}^{n} \subset X$ and $\left(f_{i}\right)_{i=1}^{n} \subset X^{\prime}$ such that $f_{i}\left(x_{j}\right)=\delta_{i, j}$ $(1 \leq i, j \leq n)$. We shall say that the biorthogonal system $\left\{y_{i}, g_{i}\right\}_{i=1}^{n}$ is normalized if $\left(y_{i}\right)_{i=1}^{n}$ and $\left(g_{i}\right)_{i=1}^{n}$ are normalized, that it is $C$-complemented if $\left\|\sum_{i} g_{i} \otimes y_{i}\right\| \leq C$, and that it is $C$-equivalent to a biorthogonal system $\left\{x_{i}, f_{i}\right\}_{i=1}^{n}$ if, for every scalar sequence $\left(\alpha_{i}\right)_{i=1}^{n}$,

$$
\left\|\sum_{i} \alpha_{i} y_{i}\right\| \leq C\left\|\sum_{i} \alpha_{i} x_{i}\right\| \text { and }\left\|\sum_{i} \alpha_{i} g_{i}\left|\mathcal{Y}\|\leq C\| \sum_{i} \alpha_{i} f_{i}\right| \mathcal{X}\right\|,
$$

where $\mathcal{X}=\left[x_{i}\right]_{i=1}^{n}$ and $\mathcal{Y}=\left[y_{i}\right]_{i=1}^{n}$. Note that two biorthogonal systems $\left\{x_{i}, f_{i}\right\}_{i=1}^{n}$ and $\left\{y_{i}, g_{i}\right\}_{i=1}^{n}$ are $C$-equivalent if and only if, for every scalar sequence $\left(\alpha_{i}\right)_{i=1}^{n}$,

$$
\frac{1}{C}\left\|\sum_{i} \alpha_{i} x_{i}\right\| \leq\left\|\sum_{i} \alpha_{i} y_{i}\right\| \leq C\left\|\sum_{i} \alpha_{i} x_{i}\right\| .
$$

To see this, simply note that if $T: \mathcal{X}^{\prime} \rightarrow \mathcal{Y}^{\prime}$ and $S: \mathcal{Y} \rightarrow \mathcal{X}$ are the linear maps defined by $\left.T f_{i}\right|_{\mathcal{X}}:=\left.g_{i}\right|_{\mathcal{Y}}(1 \leq i \leq n)$ and $S y_{i}:=x_{i}(1 \leq$ $i \leq n)$, respectively, then $T^{*}=S$, so $\|S\|=\|T\|$. Thus, $C$-equivalence of biorthogonal systems is a symmetric relation.

We shall call a biorthogonal system $\left\{y_{i}, g_{i}\right\}_{i=1}^{n}$ in a Banach space $X$ with Schauder basis $\left(x_{i}\right)$ a block biorthogonal system (b.b.s. for short) if the $y_{i}$ 's are disjointly supported with respect to $\left(x_{i}\right)$, each $g_{i}$ is a support functional 
for $y_{i}$ and $\operatorname{supp} g_{i}=\operatorname{supp} y_{i}(1 \leq i \leq n)$. (Note that we do not require $\operatorname{supp} y_{1}<\cdots<\operatorname{supp} y_{n}$.)

With this, we are at last ready to state and prove our main result.

TheOREM 5.5. Let $X$ be a Banach space with a u.s.m. basis $\left(x_{i}\right)$ and without non-trivial Hilbert components. Then $\mathcal{A}_{0}(X)$ is 1-amenable if and only if, given an arbitrary null sequence $\left(\varepsilon_{n}\right) \subset \mathbb{R}_{+}$, there are, for every $n \in \mathbb{N}$, normalized block biorthogonal systems $\left\{x_{j, 1}, f_{j, 1}\right\}_{j=1}^{n},\left\{x_{j, 2}, f_{j, 2}\right\}_{j=1}^{n}$, $\ldots,\left\{x_{j, N_{n}}, f_{j, N_{n}}\right\}_{j=1}^{n},\left(1+\varepsilon_{n}\right)$-complemented and $\left(1+\varepsilon_{n}\right)$-equivalent to $\left\{x_{j}, x_{j}^{*}\right\}_{j=1}^{n}$, and positive scalars $\lambda_{1}, \ldots, \lambda_{N_{n}}$ satisfying $\sum_{i} \lambda_{i}=1$, such that

$$
\sum_{k}\left|\sum_{i=1}^{N_{n}} \lambda_{i} f_{1, i}\left(x_{k}\right) x_{k}^{*}\left(x_{1, i}\right)-\sum_{i=1}^{N_{n}} \lambda_{i} f_{j, i}\left(x_{k}\right) x_{k}^{*}\left(x_{j, i}\right)\right| \leq \varepsilon_{n}
$$

for every $1<j \leq n$.

Proof. Suppose first that $\left(x_{i}\right)$ is a basis satisfying the conditions of the theorem, and let $\left(\varepsilon_{n}\right)$ be a non-negative sequence such that $\lim _{n} n \varepsilon_{n}=0$. For every $n \in \mathbb{N}$, define

$$
d_{n}:=\frac{1}{2^{n+K_{n}}} \sum_{\xi \in\{-1,1\}^{n}} \sum_{\eta \in\{-1,1\}^{K_{n}}} \sum_{i=1}^{N_{n}} \lambda_{i} U_{\xi} R_{i} U_{\eta} \otimes U_{\eta} S_{i} U_{\xi},
$$

where

$$
\begin{gathered}
K_{n}:=\max \bigcup_{\substack{1 \leq j \leq n \\
1 \leq i \leq N_{n}}} \operatorname{supp} x_{j, i}, \quad U_{\xi}:=\sum_{j=1}^{n} \xi_{j} x_{j}^{*} \otimes x_{j}, \quad U_{\eta}:=\sum_{k=1}^{K_{n}} \eta_{k} x_{k}^{*} \otimes x_{k}, \\
R_{i}:=\sum_{j=1}^{n} f_{j, i} \otimes x_{j} \quad \text { and } \quad S_{i}:=\sum_{j=1}^{n} x_{j}^{*} \otimes x_{j, i} \quad\left(1 \leq i \leq N_{n}\right),
\end{gathered}
$$

and the $x_{j, i}$ 's and $f_{j, i}$ 's have been chosen to satisfy the hypotheses of the theorem with respect to the sequence $\left(\varepsilon_{n}\right)$. We show next that the sequence $\left(d_{n}\right)$ is an approximate diagonal for $\mathcal{A}_{0}(X)$ such that $\left\|d_{n}\right\|_{\wedge} \leq\left(1+\varepsilon_{n}\right)^{3}$ $(n \in \mathbb{N})$.

It follows easily from our definition of $d_{n}$ that

$$
\pi\left(d_{n}\right)=\sum_{j=1}^{n} x_{j}^{*} \otimes x_{j}=: P_{n} .
$$

Let

$$
\widetilde{d}_{n}:=\sum_{\substack{1 \leq j \leq n \\ 1 \leq k \leq K_{n}}}\left(\sum_{i=1}^{N_{n}} \lambda_{i} f_{1, i}\left(x_{k}\right) x_{k}^{*}\left(x_{1, i}\right)\right)\left(x_{k}^{*} \otimes x_{j}\right) \otimes\left(x_{j}^{*} \otimes x_{k}\right) \quad(n \in \mathbb{N}),
$$


so $\widetilde{d}_{n}$ is a generalized diagonal for $\mathcal{F}\left(X_{n}\right)$. Then note that

$$
\begin{aligned}
d_{n} & =\frac{1}{2^{n+K_{n}}} \sum_{\xi} \sum_{\eta} \sum_{i=1}^{N_{n}} \lambda_{i}\left(\sum_{h=1}^{n} f_{h, i} U_{\eta} \otimes \xi_{h} x_{h}\right) \otimes\left(\sum_{j=1}^{n} \xi_{j} x_{j}^{*} \otimes U_{\eta} x_{j, i}\right) \\
& =\frac{1}{2^{K_{n}}} \sum_{\eta} \sum_{i=1}^{N_{n}} \lambda_{i} \sum_{1 \leq h, j \leq n}\left(\frac{1}{2^{n}} \sum_{\xi} \xi_{j} \xi_{h}\right)\left(f_{h, i} U_{\eta} \otimes x_{h}\right) \otimes\left(x_{j}^{*} \otimes U_{\eta} x_{j, i}\right) \\
& =\sum_{i=1}^{N_{n}} \lambda_{i} \sum_{1 \leq j \leq n} \sum_{1 \leq k, l \leq K_{n}}\left(\frac{1}{2^{K_{n}}} \sum_{\eta} \eta_{k} \eta_{l}\right)\left(f_{j, i}\left(x_{k}\right) x_{k}^{*} \otimes x_{j}\right) \otimes\left(x_{j}^{*} \otimes x_{l}^{*}\left(x_{j, i}\right) x_{l}\right) \\
& =\sum_{\substack{1 \leq j \leq n \\
1 \leq k \leq K_{n}}}\left(\sum_{i=1}^{N_{n}} \lambda_{i} f_{j, i}\left(x_{k}\right) x_{k}^{*}\left(x_{j, i}\right)\right)\left(x_{k}^{*} \otimes x_{j}\right) \otimes\left(x_{j}^{*} \otimes x_{k}\right) .
\end{aligned}
$$

By $5.2,\left\|\widetilde{d}_{n}-d_{n}\right\|_{\wedge} \leq n \varepsilon_{n}$, and so, for every $W \in \mathcal{F}\left(X_{n}\right)$,

$$
\left\|W d_{n}-d_{n} W\right\|_{\wedge} \leq\|W\|\left\|d_{n}-\widetilde{d}_{n}\right\|_{\wedge}+\left\|\widetilde{d}_{n}-d_{n}\right\|_{\wedge}\|W\| \leq 2 n \varepsilon_{n}\|W\| .
$$

Since $\mathcal{A}_{0}(X)$ is the inductive limit of the algebras $\mathcal{F}\left(X_{n}\right)$ we must have $P_{n} W P_{n} \rightarrow W\left(W \in \mathcal{A}_{0}(X)\right)$. Combining this fact with the previous inequality one easily concludes that $W d_{n}-d_{n} W \rightarrow 0\left(W \in \mathcal{A}_{0}(X)\right)$.

Lastly, as $\left\{x_{j, i}, f_{j, i}\right\}_{j=1}^{n}$ is $\left(1+\varepsilon_{n}\right)$-complemented and $\left(1+\varepsilon_{n}\right)$-equivalent to $\left\{x_{j}, x_{j}^{*}\right\}_{j=1}^{n}$, one has

$$
\begin{aligned}
\left\|R_{i}(x)\right\| & =\left\|\sum_{j} f_{j, i}(x) x_{j}\right\| \\
& \leq\left(1+\varepsilon_{n}\right)\left\|\sum_{j} f_{j, i}(x) x_{j, i}\right\| \leq\left(1+\varepsilon_{n}\right)^{2}\|x\| \quad(x \in X),
\end{aligned}
$$

and also

$$
\begin{aligned}
\left\|S_{i}(x)\right\| & =\left\|\sum_{j} x_{j}^{*}(x) x_{j, i}\right\| \\
& \leq\left(1+\varepsilon_{n}\right)\left\|\sum_{j} x_{j}^{*}(x) x_{j}\right\| \leq\left(1+\varepsilon_{n}\right)\|x\| \quad(x \in X),
\end{aligned}
$$

so

$$
\left\|d_{n}\right\|_{\wedge}=\sum_{i} \lambda_{i}\left\|R_{i}\right\|\left\|S_{i}\right\| \leq \max _{i}\left\|R_{i}\right\| \max _{i}\left\|S_{i}\right\| \leq\left(1+\varepsilon_{n}\right)^{3} .
$$

Thus, if $\left(x_{i}\right)$ satisfies the conditions of the theorem then $\mathcal{A}_{0}(X)$ is 1-amenable.

Conversely, suppose $\mathcal{A}_{0}(X)$ is 1-amenable and let $\left(\varepsilon_{n}\right)$ be a positive sequence bounded by 1 and converging to zero. (Note that the general case, in which $\left(\varepsilon_{n}\right)$ is an arbitrary positive sequence converging to zero, follows im- 
mediately from this one.) For each $\varepsilon_{n}$, choose $0<\tau_{n}<\varepsilon_{n}^{2} /(30 n)^{2}$ such that for every pair of norm-one linear maps $V: X_{n} \rightarrow X_{m}$ and $W$ : $X_{m} \rightarrow X_{n}$ satisfying $\left\|V W-P_{n}\right\| \leq \tau_{n}$ there are disjoint subsets $E_{1}, \ldots, E_{n}$ of $\{1, \ldots, m\}$ such that $\left\|W x_{j}-E_{j} W x_{j}\right\| \leq \varepsilon_{n}^{2} /(30 n)^{2}(1 \leq j \leq n)$, which is possible by Lemma 5.4 and the uniform strict monotonicity of the basis $\left(x_{i}\right)$. Next, let $\varkappa\left(\tau_{n}\right)$ be as in Lemma 5.2, and choose $\delta_{n}>0$ small enough so that $8\left(1+2 \varkappa\left(\tau_{n}\right)\right) \delta_{n} \leq \varepsilon_{n}$. Lastly, choose a bounded approximate diagonal $\left(d_{n}\right)_{n \in \mathbb{N}}$ for $\mathcal{A}_{0}(X)$ such that

(i) $d_{n} \in \mathcal{F}\left(X_{K_{n}}, X_{n}\right) \widehat{\otimes} \mathcal{F}\left(X_{n}, X_{K_{n}}\right)$ for some $K_{n} \in \mathbb{N}$;

(ii) $\pi\left(d_{n}\right)=P_{n}$;

(iii) $W d_{n}=d_{n} W\left(W \in P_{n} \mathcal{A}(X) P_{n}\right)$; and

(iv) $\left\|d_{n}\right\|_{\wedge} \leq 1+\delta_{n}$.

As indicated in Section 2, the proof that such an approximate diagonal exists is exactly the same as that of [BG2, Proposition 3.1].

Now, fix $n$. As in the proof of Theorem 4.4, the finite-dimensionality of $X_{n}$ and $X_{K_{n}}$ implies that $d_{n}$ admits a finite representation $\sum_{i=1}^{M_{n}} R_{i} \otimes S_{i}$ such that $\sum_{i=1}^{M_{n}} R_{i} S_{i}=P_{n}$ and $\sum_{i=1}^{M_{n}}\left\|R_{i}\right\|\left\|S_{i}\right\| \leq 1+\delta_{n}$. We shall assume that $\left\|R_{i}\right\|\left\|S_{i}\right\| \neq 0\left(1 \leq i \leq M_{n}\right)$. By Lemma 5.2 ,

$$
\sum_{i \in I_{\tau_{n}}}\left\|R_{i}\right\|\left\|S_{i}\right\| \leq \varkappa\left(\tau_{n}\right) \delta_{n}
$$

where

$$
I_{\tau_{n}}=\left\{i:\left\|\left(\left\|R_{i}\right\|^{-1} R_{i}\right)\left(\left\|S_{i}\right\|^{-1} S_{i}\right)-P_{n}\right\|>\tau_{n}\right\} .
$$

For each $1 \leq i \leq M_{n}$, let $V_{i}=\left\|R_{i}\right\|^{-1} R_{i}$ and $W_{i}=\left\|S_{i}\right\|^{-1} S_{i}$. Note, in particular, that $\left\|V_{i} W_{i}-P_{n}\right\| \leq \tau_{n}\left(i \in I_{\tau_{n}}^{c}\right)$. Hence, by our choice of $\tau_{n}$, for every $i \in I_{\tau_{n}}^{c}$, there exist disjoint subsets $E_{1, i}, \ldots, E_{n, i}$ of $\left\{1, \ldots, K_{n}\right\}$ such that $\left\|W_{i} x_{j}-E_{j, i} W_{i} x_{j}\right\| \leq \varepsilon_{n}^{2} /(30 n)^{2}(1 \leq j \leq n)$. Let $y_{j, i}:=E_{j, i} W_{i} x_{j}$ and $g_{j, i}:=x_{j}^{*} V_{i} E_{j, i}\left(i \in I_{\tau_{n}}^{c}, 1 \leq j \leq n\right)$. Then

$$
\begin{aligned}
\left|1-g_{j, i}\left(y_{j, i}\right)\right| & \leq\left|x_{j}^{*}\left(x_{j}\right)-x_{j}^{*} V_{i}\left(W_{i} x_{j}\right)\right|+\left|x_{j}^{*} V_{i}\left(W_{i} x_{j}-y_{j, i}\right)\right| \\
& \leq \tau_{n}+\left(\frac{\varepsilon_{n}}{30 n}\right)^{2} \leq 2\left(\frac{\varepsilon_{n}}{30 n}\right)^{2},
\end{aligned}
$$

and

$$
\begin{aligned}
\left|1-\left\|g_{j, i}\right\|^{-1} g_{j, i}\left(y_{j, i}\right)\right| & \\
& \leq\left|1-g_{j, i}\left(y_{j, i}\right)\right|+\frac{\left|1-\left\|g_{j, i}\right\|\right|}{\left\|g_{j, i}\right\|} \leq\left|1-g_{j, i}\left(y_{j, i}\right)\right|+\frac{\left|1-g_{j, i}\left(y_{j, i}\right)\right|}{1-\left|1-g_{j, i}\left(y_{j, i}\right)\right|} \\
& \leq 2\left(\frac{\varepsilon_{n}}{30 n}\right)^{2}+\frac{2\left(\frac{\varepsilon_{n}}{30 n}\right)^{2}}{1-2\left(\frac{\varepsilon_{n}}{30 n}\right)^{2}} \leq\left(\frac{\varepsilon_{n}}{15 n}\right)^{2} \frac{1-\frac{1}{900}}{1-\frac{2}{900}} \leq\left(\frac{\varepsilon_{n}}{14 n}\right)^{2} .
\end{aligned}
$$


By the Bishop-Phelps-Bollobás theorem ([BD, Section 16, Theorem 1]), there are pairs $\left(x_{j, i}, f_{j, i}\right) \in\left[x_{j}: j \in E_{j, i}\right] \times\left[x_{j}^{*}: j \in E_{j, i}\right]\left(i \in I_{\tau_{n}}^{c}, 1 \leq j \leq n\right)$ such that $\left\|x_{j, i}\right\|=\left\|f_{j, i}\right\|=f_{j, i}\left(x_{j, i}\right)=1,\left\|y_{j, i}-x_{j, i}\right\| \leq \varepsilon_{n} /(7 n)$ and \|\|$g_{j, i}\left\|^{-1} g_{j, i}-f_{j, i}\right\| \leq \varepsilon_{n} /(7 n)$. Note that

$$
\begin{aligned}
\left\|f_{j, i}-g_{j, i}\right\| & \leq\left\|g_{j, i}-\right\| g_{j, i}\left\|^{-1} g_{j, i}\right\|+\frac{\varepsilon_{n}}{7 n} \\
& \leq\left|1-g_{j, i}\left(y_{j, i}\right)\right|+\frac{\varepsilon_{n}}{7 n} \leq 2\left(\frac{\varepsilon_{n}}{30 n}\right)^{2}+\frac{\varepsilon_{n}}{7 n} .
\end{aligned}
$$

Then, for every scalar sequence $\alpha_{1}, \ldots, \alpha_{n}$, we have

$$
\begin{aligned}
\left\|\sum_{j} \alpha_{j} x_{j, i}\right\| & \leq \sum_{j}\left|\alpha_{j}\right|\left\|x_{j, i}-y_{j, i}\right\|+\sum_{j}\left|\alpha_{j}\right|\left\|y_{j, i}-W_{i} x_{j}\right\|+\left\|\sum_{j} \alpha_{j} W_{i} x_{j}\right\| \\
& \leq\left(\frac{\varepsilon_{n}}{7 n}+\left(\frac{\varepsilon_{n}}{30 n}\right)^{2}\right) \sum_{j}\left|\alpha_{j}\right|+\left\|\sum_{j} \alpha_{j} x_{j}\right\| \\
& \leq\left(1+\frac{\varepsilon_{n}}{7}+\frac{\varepsilon_{n}^{2}}{900 n}\right)\left\|\sum_{j} \alpha_{j} x_{j}\right\|,
\end{aligned}
$$

and

$$
\begin{aligned}
\left(1-\tau_{n}\right) & \left\|\sum_{j} \alpha_{j} x_{j}\right\| \\
& \leq\left\|V_{i} W_{i}\left(\sum_{j} \alpha_{j} x_{j}\right)\right\| \leq\left\|\sum_{j} \alpha_{j} W_{i} x_{j}\right\| \\
& \leq \sum_{j}\left|\alpha_{j}\right|\left\|W_{i} x_{j}-y_{j, i}\right\|+\sum_{j}\left|\alpha_{j}\right|\left\|y_{j, i}-x_{j, i}\right\|+\left\|\sum_{j} \alpha_{j} x_{j, i}\right\| \\
& \leq\left(1+\frac{\varepsilon_{n}}{7}+\frac{\varepsilon_{n}^{2}}{900 n}\right)\left\|\sum_{j} \alpha_{j} x_{j, i}\right\|,
\end{aligned}
$$

which in turn, since $\varepsilon_{n} \rightarrow 0$, yields

$$
\frac{1}{1+\varepsilon_{n}}\left\|\sum_{j} \alpha_{j} x_{j}\right\| \leq\left\|\sum_{j} \alpha_{j} x_{j, i}\right\| \leq\left(1+\varepsilon_{n}\right)\left\|\sum_{j} \alpha_{j} x_{j}\right\| .
$$

Thus, each b.b.s. $\left\{x_{j, i}, f_{j, i}\right\}_{j=1}^{n}\left(i \in I_{\tau_{n}}^{c}\right)$ is $\left(1+\varepsilon_{n}\right)$-equivalent to $\left\{x_{j}, x_{j}^{*}\right\}_{j=1}^{n}$.

To show that each b.b.s. $\left\{x_{j, i}, f_{j, i}\right\}_{j=1}^{n}\left(i \in I_{\tau_{n}}^{c}\right)$ is $\left(1+\varepsilon_{n}\right)$-complemented, we argue as follows. First, for every $\xi \in\{-1,1\}^{n}$, define $E_{i, \xi}:=\sum_{j} \xi_{j} E_{j, i}$. Then define $\Phi_{i, \xi}: X^{\prime} \otimes X \rightarrow X^{\prime} \otimes X$ by

$$
\Phi_{i, \xi}(f \otimes x):=f V_{i} E_{i, \xi} \otimes E_{i, \xi} W_{i} x,
$$

and define $\Phi_{i}:=2^{-n} \sum_{\xi} \Phi_{i, \xi}$. Note that each $\Phi_{i, \xi}$ has norm $\left\|V_{i}\right\|\left\|W_{i}\right\| \leq 1$, 
so $\left\|\Phi_{i}\right\| \leq 1$. We then have

$$
\begin{aligned}
1 \geq & \left\|\Phi_{i}\left(\sum_{j} x_{j}^{*} \otimes x_{j}\right)\right\| \\
= & \left\|\sum_{j} \sum_{k, l} 2^{-n}\left(\sum_{\xi} \xi_{k} \xi_{l}\right) x_{j}^{*} V_{i} E_{k, i} \otimes E_{l, i} W_{i} x_{j}\right\| \\
= & \left\|\sum_{j} \sum_{k} x_{j}^{*} V_{i} E_{k, i} \otimes E_{k, i} W_{i} x_{j}\right\| \\
\geq & \left\|\sum_{j} g_{j, i} \otimes y_{j, i}\right\|-\sum_{k \neq j}\left\|E_{k, i} W_{i} x_{j}\right\| \\
\geq & \left\|\sum_{j} f_{j, i} \otimes x_{j, i}\right\|-\sum_{j}\left\|f_{j, i}-g_{j, i}\right\| \\
& -\sum_{j}\left\|x_{j, i}-y_{j, i}\right\|-(n-1) \sum_{j}\left\|\left(\mathrm{id}-E_{j, i}\right) W_{i} x_{j}\right\|,
\end{aligned}
$$

which combined with our previous estimates gives

$$
\begin{aligned}
& \left\|\sum_{j} f_{j, i} \otimes x_{j, i}\right\| \\
& \quad \leq 1+n\left(\frac{\varepsilon_{n}}{7 n}\right)+n\left(\frac{\varepsilon_{n}}{7 n}+2\left(\frac{\varepsilon_{n}}{30 n}\right)^{2}\right)+n^{2}\left(\frac{\varepsilon_{n}}{30 n}\right)^{2} \leq 1+\varepsilon_{n},
\end{aligned}
$$

as required.

To finish our proof we show that

$$
\sum_{k}\left|\sum_{i \in I_{\tau_{n}}^{c}} \lambda_{i} f_{1, i}\left(x_{k}\right) x_{k}^{*}\left(x_{1, i}\right)-\sum_{i \in I_{\tau_{n}}^{c}} \lambda_{i} f_{j, i}\left(x_{k}\right) x_{k}^{*}\left(x_{j, i}\right)\right| \leq \varepsilon_{n} \quad(1 \leq j \leq n) .
$$

So $N_{n}=\left|I_{\tau_{n}}^{c}\right|$.

First, the fact that $d_{n}$ is a generalized diagonal for $\mathcal{F}\left(X_{n}\right)$ easily implies that

$$
\left(x_{1}^{*} \otimes x_{k}\right) d_{n}\left(x_{k}^{*} \otimes x_{1}\right)=\left(x_{j}^{*} \otimes x_{k}\right) d_{n}\left(x_{k}^{*} \otimes x_{j}\right) \quad\left(1<j \leq n, 1 \leq k \leq K_{n}\right) .
$$

Applying the linear functional $\mathcal{F}(X) \otimes \mathcal{F}(X) \rightarrow \mathbb{C}, R \otimes S \mapsto \operatorname{tr}(R) \operatorname{tr}(S)$, on both sides of the last identity one obtains

$$
\sum_{i=1}^{M_{n}} x_{1}^{*}\left(R_{i} x_{k}\right) x_{k}^{*}\left(S_{i} x_{1}\right)=\sum_{i=1}^{M_{n}} x_{j}^{*}\left(R_{i} x_{k}\right) x_{k}^{*}\left(S_{i} x_{j}\right),
$$

or equivalently,

$$
\sum_{i=1}^{M_{n}} \widetilde{\lambda}_{i} x_{1}^{*}\left(V_{i} x_{k}\right) x_{k}^{*}\left(W_{i} x_{1}\right)=\sum_{i=1}^{M_{n}} \widetilde{\lambda}_{i} x_{j}^{*}\left(V_{i} x_{k}\right) x_{k}^{*}\left(W_{i} x_{j}\right) \quad(1<j \leq n),
$$


where $\widetilde{\lambda}_{i}=\left\|R_{i}\right\|\left\|S_{i}\right\|\left(1 \leq i \leq M_{n}\right)$. Define $\lambda_{i}:=\widetilde{\lambda}_{i} / \sum_{l \in I_{\tau_{n}}^{c}} \widetilde{\lambda}_{l}\left(i \in I_{\tau_{n}}^{c}\right)$. Using (5.4), one obtains

$$
\begin{aligned}
\left|\sum_{i \in I_{\tau_{n}}^{c}} \lambda_{i} f_{1, i}\left(x_{k}\right) x_{k}^{*}\left(x_{1, i}\right)-\sum_{i \in I_{\tau_{n}}^{c}} \lambda_{i} f_{j, i}\left(x_{k}\right) x_{k}^{*}\left(x_{j, i}\right)\right| \\
\leq \sum_{i \in I_{\tau_{n}}^{c}}\left|\lambda_{i} f_{1, i}\left(x_{k}\right) x_{k}^{*}\left(x_{1, i}\right)-\widetilde{\lambda}_{i} x_{1}^{*}\left(V_{i} x_{k}\right) x_{k}^{*}\left(W_{i} x_{1}\right)\right| \\
\quad+\sum_{i \in I_{\tau_{n}}^{c}}\left|\widetilde{\lambda}_{i} x_{j}^{*}\left(V_{i} x_{k}\right) x_{k}^{*}\left(W_{i} x_{j}\right)-\lambda_{i} f_{j, i}\left(x_{k}\right) x_{k}^{*}\left(x_{j, i}\right)\right| \\
\quad+\sum_{i \in I_{\tau_{n}}} \widetilde{\lambda}_{i}\left|x_{1}^{*}\left(V_{i} x_{k}\right) x_{k}^{*}\left(W_{i} x_{1}\right)\right|+\sum_{i \in I_{\tau_{n}}} \widetilde{\lambda}_{i}\left|x_{j}^{*}\left(V_{i} x_{k}\right) x_{k}^{*}\left(W_{i} x_{j}\right)\right| .
\end{aligned}
$$

For each $1 \leq j \leq n$, choose $\eta_{j} \in \mathbb{T}^{K_{n}}$ (where $\left.\mathbb{T}=\{z \in \mathbb{C}:|z|=1\}\right)$ such that

$$
\begin{aligned}
\sum_{k} \mid \lambda_{i} f_{j, i}\left(x_{k}\right) x_{k}^{*}\left(x_{j, i}\right)-\tilde{\lambda}_{i} x_{j}^{*}( & \left.V_{i} x_{k}\right) x_{k}^{*}\left(W_{i} x_{j}\right) \mid \\
= & \left|\lambda_{i} f_{j, i}\left(U_{\eta_{j}} x_{j, i}\right)-\widetilde{\lambda}_{i} x_{j}^{*}\left(V_{i} U_{\eta_{j}} W_{i} x_{j}\right)\right| .
\end{aligned}
$$

Then note that

$$
\begin{aligned}
\mid f_{j, i}\left(U_{\eta_{j}} x_{j, i}\right)- & x_{j}^{*}\left(V_{i} U_{\eta_{j}} W_{i} x_{j}\right) \mid \\
\leq & \left|f_{j, i}\left(U_{\eta_{j}} x_{j, i}\right)-f_{j, i}\left(U_{\eta_{j}} y_{j, i}\right)\right|+\left|f_{j, i}\left(U_{\eta_{j}} y_{j, i}\right)-x_{j}^{*}\left(V_{i} U_{\eta_{j}} y_{j, i}\right)\right| \\
& \quad+\left|x_{j}^{*}\left(V_{i} U_{\eta_{j}} y_{j, i}\right)-x_{j}^{*}\left(V_{i} U_{\eta_{j}} W_{i} x_{j}\right)\right| \\
\leq & \left\|x_{j, i}-y_{j, i}\right\|+\left\|f_{j, i}-g_{j, i}\right\|+\left\|y_{j, i}-W_{i} x_{j}\right\| \leq \frac{2 \varepsilon_{n}}{7 n}+3\left(\frac{\varepsilon_{n}}{30 n}\right)^{2} .
\end{aligned}
$$

Combining this last estimate with the previous identity and taking 5.3$)$ into account we obtain, for every $1 \leq j \leq n$,

$$
\begin{aligned}
& \sum_{k}\left|\lambda_{i} f_{j, i}\left(x_{k}\right) x_{k}^{*}\left(x_{j, i}\right)-\widetilde{\lambda}_{i} x_{j}^{*}\left(V_{i} x_{k}\right) x_{k}^{*}\left(W_{i} x_{j}\right)\right| \\
& \quad \leq \lambda_{i}\left|f_{j, i}\left(U_{\eta_{j}} x_{j, i}\right)-x_{j}^{*}\left(V_{i} U_{\eta_{j}} W_{i} x_{j}\right)\right|+\left|\lambda_{i}-\widetilde{\lambda}_{i}\right|\left|x_{j}^{*}\left(V_{i} U_{\eta_{j}} W_{i} x_{j}\right)\right| \\
& \quad \leq \lambda_{i}\left(\frac{2 \varepsilon_{n}}{7 n}+3\left(\frac{\varepsilon_{n}}{30 n}\right)^{2}\right)+\lambda_{i}\left|1-\sum_{l \in I_{\tau_{n}}^{c}} \widetilde{\lambda}_{l}\right| \\
& \quad \leq \lambda_{i}\left(\frac{2 \varepsilon_{n}}{7 n}+3\left(\frac{\varepsilon_{n}}{30 n}\right)^{2}+\left(1+\varkappa\left(\tau_{n}\right)\right) \delta_{n}\right) .
\end{aligned}
$$

Similarly, for every $1 \leq j \leq n$, there is a $\zeta_{j} \in \mathbb{T}^{K_{n}}$ such that

$$
\sum_{k}\left|x_{j}^{*}\left(V_{i} x_{k}\right) x_{k}^{*}\left(W_{i} x_{j}\right)\right|=\left|x_{j}^{*}\left(V_{i} U_{\zeta_{j}} W_{i} x_{j}\right)\right| \leq 1 .
$$


Finally, combining (5.6) and (5.7) with (5.5), and once again taking into account 5.3, we obtain

$$
\begin{aligned}
\sum_{k} \mid \sum_{i \in I_{\tau_{n}}^{c}} & \lambda_{i} f_{1, i}\left(x_{k}\right) x_{k}^{*}\left(x_{1, i}\right)-\sum_{i \in I_{\tau_{n}}^{c}} \lambda_{i} f_{j, i}\left(x_{k}\right) x_{k}^{*}\left(x_{j, i}\right) \mid \\
\leq & 2 \sum_{i \in I_{\tau_{n}}^{c}} \lambda_{i}\left(\frac{2 \varepsilon_{n}}{7 n}+3\left(\frac{\varepsilon_{n}}{30 n}\right)^{2}+\left(1+\varkappa\left(\tau_{n}\right)\right) \delta_{n}\right)+2 \sum_{i \in I_{\tau_{n}}} \widetilde{\lambda}_{i} \\
\leq & \frac{4 \varepsilon_{n}}{7 n}+6\left(\frac{\varepsilon_{n}}{30 n}\right)^{2}+2\left(1+2 \varkappa\left(\tau_{n}\right)\right) \delta_{n} \\
\leq & \frac{4 \varepsilon_{n}}{7 n}+6\left(\frac{\varepsilon_{n}}{30 n}\right)^{2}+\frac{\varepsilon_{n}}{4}<\varepsilon_{n} .
\end{aligned}
$$

This concludes the proof of the theorem.

REMARK 5.6. It might be worth pointing out that condition $(5.2)$ is equivalent to the following:

$$
\max _{\eta \in\{-1,1\}^{\mathbb{N}}}\left\{\sum_{i=1}^{N_{n}} \lambda_{i} f_{1, i}\left(U_{\eta} x_{1, i}\right)-\sum_{i=1}^{N_{n}} \lambda_{i} f_{j, i}\left(U_{\eta} x_{j, i}\right)\right\} \leq \varepsilon_{n} \quad(1<j \leq n) .
$$

Indeed, since each $f_{j, i}$ is a support functional for $x_{j, i}$ and the basis $\left(x_{i}\right)$ is 1-unconditional, there is $\eta \in\{-1,1\}^{\mathbb{N}}$ such that

$$
\begin{aligned}
\sum_{k}\left|\sum_{i=1}^{N_{n}} \lambda_{i} f_{1, i}\left(x_{k}\right) x_{k}^{*}\left(x_{1, i}\right)-\sum_{i=1}^{N_{n}} \lambda_{i} f_{j, i}\left(x_{k}\right) x_{k}^{*}\left(x_{j, i}\right)\right| \\
=\sum_{k} \eta_{k}\left(\sum_{i=1}^{N_{n}} \lambda_{i} f_{1, i}\left(x_{k}\right) x_{k}^{*}\left(x_{1, i}\right)-\sum_{i=1}^{N_{n}} \lambda_{i} f_{j, i}\left(x_{k}\right) x_{k}^{*}\left(x_{j, i}\right)\right) \\
=\sum_{i=1}^{N_{n}} \lambda_{i} f_{1, i}\left(U_{\eta} x_{1, i}\right)-\sum_{i=1}^{N_{n}} \lambda_{i} f_{j, i}\left(U_{\eta} x_{j, i}\right) \\
\leq \max _{\xi \in\{-1,1\}^{\mathbb{N}}}\left\{\sum_{i=1}^{N_{n}} \lambda_{i} f_{1, i}\left(U_{\xi} x_{1, i}\right)-\sum_{i=1}^{N_{n}} \lambda_{i} f_{j, i}\left(U_{\xi} x_{j, i}\right)\right\},
\end{aligned}
$$

and the opposite inequality is proved similarly. Notice also that since the basis $\left(x_{i}\right)$ is 1-unconditional the block biorthogonal systems of Theorem 5.5 can be chosen to be positive, i.e., with each vector in them having all its coordinates with respect to either $\left(x_{i}\right)$ or $\left(x_{i}^{*}\right)$ real positive.

In the following two examples, the corresponding algebras of approximable operators are well known to be amenable. Their only purpose is to illustrate the conditions of Theorem 5.5 in some concrete situations. 
EXAMPLE 5.7. Let $X$ be a Banach space with a normalized 1-unconditional basis $\left(x_{i}\right)$ such that the left and right shift operators with respect to it are both norm-one. Then, for every null sequence $\left(\varepsilon_{n}\right)$ there is a sequence $\left(N_{n}\right)$ of positive integers such that, for every $n \in \mathbb{N}$, the block biorthogonal systems $\left\{x_{i+j}, x_{i+j}^{*}\right\}_{j=1}^{n}, 0 \leq i \leq N_{n}-1$, satisfy the conditions of Theorem 5.5 with respect to the given sequence $\left(\varepsilon_{n}\right)$. Indeed, each b.b.s. $\left\{x_{i+j}, x_{i+j}^{*}\right\}_{j=1}^{n}$ is clearly 1-complemented and 1-equivalent to $\left\{x_{j}, x_{j}^{*}\right\}_{j=1}^{n}$ (the latter because both shifts are norm-one). As for (5.2), note that

$$
\begin{aligned}
\sum_{k} \mid \frac{1}{N} \sum_{i=0}^{N-1} x_{i+1}^{*}\left(x_{k}\right) x_{k}^{*}\left(x_{i+1}\right) & -\frac{1}{N} \sum_{i=0}^{N-1} x_{i+j}^{*}\left(x_{k}\right) x_{k}^{*}\left(x_{i+j}\right) \mid \\
& =\frac{1}{N} \sum_{k}\left|\sum_{i=1}^{N} \delta_{i, k}-\sum_{i=j}^{N+j-1} \delta_{i, k}\right| \\
& =\frac{1}{N} \sum_{k}\left|\sum_{i=1}^{j-1} \delta_{i, k}-\sum_{i=N+1}^{N+j-1} \delta_{i, k}\right| \\
& =2 \frac{j-1}{N} \quad(1 \leq j \leq n, n \in \mathbb{N}, N \in \mathbb{N}) .
\end{aligned}
$$

So, to have (5.2) satisfied with respect to $\left(\varepsilon_{n}\right)$, it is enough to choose $N_{n} \geq$ $2 n / \varepsilon_{n}(n \in \mathbb{N})$. Clearly, this will not affect the other two conditions.

ExAmple 5.8. Let $X:=\left(\bigoplus_{i} \ell_{p}^{i}\right)_{\ell_{q}}, 1 \leq p \neq q \leq \infty$, and let $\left(\varepsilon_{n}\right) \subset \mathbb{R}_{+}$ be an arbitrary null sequence. It is convenient to write down the unit vector basis of $X$ in the form $x_{1}^{(1)}, x_{1}^{(2)}, x_{2}^{(2)}, \ldots, x_{1}^{(n)}, x_{2}^{(n)}, \ldots, x_{n}^{(n)}, \ldots$, where $x_{j}^{(i)}$ $(1 \leq j \leq i, i \in \mathbb{N})$ stands for the $j$ th vector of the unit vector basis of the $i$ th summand of $X$. To simplify notations let us denote by $f_{j}^{(i)}$ the associated biorthogonal functional. Then, for every $n \in \mathbb{N}$, there is a positive integer $N_{n}$ such that the block biorthogonal systems

$$
\begin{aligned}
\left\{x_{j}^{(i)}, x_{j}^{(i+1)}, x_{j+1}^{(i+1)}, \ldots, x_{j}^{(i+n)}, x_{j+1}^{(i+n)}, \ldots, x_{j+n}^{(i+n)},\right. & \\
\left.f_{j}^{(i)}, f_{j}^{(i+1)}, f_{j+1}^{(i+1)}, \ldots, f_{j}^{(i+n)}, f_{j+1}^{(i+n)}, \ldots, f_{j+n}^{(i+n)}\right\} & \quad\left(1 \leq j \leq i, 1 \leq i \leq N_{n}\right)
\end{aligned}
$$

are 1-complemented, 1-equivalent to

$$
\begin{aligned}
& \left\{x_{1}^{(1)}, x_{1}^{(2)}, x_{2}^{(2)}, \ldots, x_{1}^{(n+1)}, x_{2}^{(n+1)}, \ldots, x_{n+1}^{(n+1)},\right. \\
& \left.\quad f_{1}^{(1)}, f_{1}^{(2)}, f_{2}^{(2)}, \ldots, f_{1}^{(n+1)}, f_{2}^{(n+1)}, \ldots, f_{n+1}^{(n+1)}\right\},
\end{aligned}
$$

and satisfy (5.2). Again, only this last needs a proof. For this, note that, in the present situation, the left hand side of (5.2) takes the form 


$$
\begin{aligned}
\frac{2}{N_{n}\left(N_{n}+1\right)} \sum_{l \in \mathbb{N}} \sum_{k=1}^{l} \mid \sum_{i=1}^{N_{n}} \sum_{j=1}^{i} f_{j}^{(i)}\left(x_{k}^{(l)}\right) f_{k}^{(l)}\left(x_{j}^{(i)}\right) & \\
& -\sum_{i=\varkappa+1}^{N_{n}+\varkappa} \sum_{j=m+1}^{i-\varkappa+m} f_{j}^{(i)}\left(x_{k}^{(l)}\right) f_{k}^{(l)}\left(x_{j}^{(i)}\right) \mid,
\end{aligned}
$$

where $0 \leq m \leq \varkappa$ and $0 \leq \varkappa \leq n$. After some straightforward simplifications this last expression becomes

$$
\begin{aligned}
& \frac{2}{N_{n}\left(N_{n}+1\right)} \sum_{l \in \mathbb{N}} \sum_{k=1}^{l} \mid \sum_{i=1}^{\varkappa} \sum_{j=1}^{i} \delta_{i, l} \delta_{j, k}+\sum_{i=\varkappa+1}^{N_{n}} \sum_{j=1}^{m} \delta_{i, l} \delta_{j, k} \\
& \quad+\sum_{i=\varkappa+1}^{N_{n}} \sum_{j=i-\varkappa+m+1}^{i} \delta_{i, l} \delta_{j, k}-\sum_{i=N_{n}+1}^{N_{n}+\varkappa} \sum_{j=m+1}^{i-\varkappa+m} \delta_{i, l} \delta_{j, k} \mid \\
& \leq \frac{2}{N_{n}\left(N_{n}+1\right)}\left[\varkappa^{2}+(\varkappa-1)\left(N_{n}-\varkappa\right)+\varkappa N_{n}\right] \leq \frac{4 n}{N_{n}+1},
\end{aligned}
$$

which can clearly be made smaller than any $\varepsilon>0$ by choosing $N_{n}$ large enough.

With this, we have verified the conditions of the theorem for every $n$ of the form $k(k+1) / 2(k \in \mathbb{N})$. But this is enough, for, in general, if the conditions of Theorem 5.5 are satisfied for every positive integer in an increasing sequence $\left(n_{k}\right)$ with respect to some null sequence $\left(\varepsilon_{k}\right) \subset \mathbb{R}_{+}$ and $n_{k-1}<m<n_{k}$ for some $k>1$, then the block biorthogonal systems $\left\{x_{j, 1}, f_{j, 1}\right\}_{j=1}^{m}, \ldots,\left\{x_{j, N_{n_{k}}}, f_{j, N_{n_{k}}}\right\}_{j=1}^{m}$, obtained from those corresponding to $n_{k}$ by removing the last $n_{k}-m$ vectors, will be $\left(1+\varepsilon_{k}\right)$-equivalent to $\left\{x_{j}, x_{j}^{*}\right\}_{j=1}^{m},\left(1+\varepsilon_{k}\right)$-complemented and still satisfy 5.2 for $\varepsilon_{k}$. From this observation one easily obtains the desired conclusion. We leave the details to the reader.

We should notice that the same kind of argument can be used for any Banach space of the form $X=\left(\bigoplus_{i} \ell_{p}^{n_{i}}\right)_{\ell_{q}}$, where $1 \leq p \neq q \leq \infty$ and $\lim _{i} n_{i}=\infty$. But, in the general case, the details are a bit more involved.

We finish the section with a few observations. First of all, note that uniform strict monotonicity of the basis is not a necessary condition for 1 -amenability. For instance, $\mathcal{A}\left(\ell_{\infty}\right)$ is 1 -amenable, but the unit vector basis of $\ell_{\infty}$ is not u.s.m. (recall our convention about the meaning of $\ell_{\infty}$ ). We do not know whether the result of Theorem 5.5 (or a similar one) will still hold if one removes this assumption.

Second, it is apparent that our proof of Theorem 5.5 relies on a 'good' description of the norm-one projections on $X$ in terms of the unconditional basis $\left(x_{i}\right)$. Unfortunately, the only results of this kind that the author is aware of depend heavily on the assumption that the unconditionality constant of 
the basis is 1 (see, for instance, the survey [Ra2]). So Theorem 5.5 may well be the best one can hope for regarding translation of amenability into a basis property.

Lastly, it seems very unlikely to the author that, even for Banach spaces with u.s.m. bases, the conditions of Theorem 5.5 can be equivalent to property $\mathbb{A}$, but we do not have any relevant example. In the next section, we shall say more about this problem. Here, just to give an idea of the sort of difficulty involved, let us look at a simple case in which 1-amenability implies property $\mathbb{A}$. First, suppose $d \in \mathcal{F}(Y, X) \widehat{\otimes} \mathcal{F}(X, Y)$ is a norm-one generalized diagonal such that $w d^{\circ}=d^{\circ} w(w \in \mathcal{F}(Y))$. This implies, in particular, that $\pi\left(d^{\circ}\right)$ is in the center of $\mathcal{F}(Y)$, i.e., it is a multiple of $\operatorname{id}_{Y}$. Let $\sum_{i=1}^{m} \lambda_{i} R_{i} \otimes S_{i}$ be a finite representation for $d$ such that $\sum_{i} \lambda_{i}=1$ and $\left\|R_{i}\right\|=\left\|S_{i}\right\|=1$ $(1 \leq i \leq m)$. From Lemma 4.2 we know that $R_{i} S_{i}=\mathrm{id}_{X}$ for every $i$. Define $\Phi: \mathcal{F}(Y, X) \widehat{\otimes} \mathcal{F}(X, Y) \rightarrow \mathcal{F}(X) \widehat{\otimes} \mathcal{F}(X)$ by

$$
\Phi(U \otimes V):=U S_{1} \otimes R_{1} V \quad(U \otimes V \in \mathcal{F}(Y, X) \widehat{\otimes} \mathcal{F}(X, Y)) .
$$

Then note that

$$
\begin{gathered}
w d=d w \Rightarrow w \Phi(d)=\Phi(d) w \quad(w \in \mathcal{F}(X)), \\
w \Phi(d)^{\circ}=w R_{1} d^{\circ} S_{1}=R_{1}\left(S_{1} w R_{1}\right) d^{\circ} S_{1} \\
=R_{1} d^{\circ}\left(S_{1} w R_{1}\right) S_{1}=R_{1} d^{\circ} S_{1} w=\Phi(d)^{\circ} w \quad(w \in \mathcal{F}(X)), \\
\pi\left(\Phi(d)^{\circ}\right)=R_{1} \pi\left(d^{\circ}\right) S_{1}=R_{1}\left(\frac{\operatorname{tr}(\pi(d))}{\operatorname{dim} Y} \operatorname{id}_{Y}\right) S_{1}=\frac{\operatorname{dim} X}{\operatorname{dim} Y} \operatorname{id}_{X},
\end{gathered}
$$

and

$$
\pi(\Phi(d))=\frac{\operatorname{tr}(\pi(\Phi(d)))}{\operatorname{dim} X} \operatorname{id}_{X}=\frac{\operatorname{tr}\left(\pi\left(\Phi(d)^{\circ}\right)\right)}{\operatorname{dim} X} \operatorname{id}_{X}=\frac{\operatorname{dim} X}{\operatorname{dim} Y} \operatorname{id}_{X},
$$

so $\Delta:=(\operatorname{dim} Y / \operatorname{dim} X) \Phi(d)$ is the corresponding symmetric diagonal for $\mathcal{F}(X)$ and $\|\Delta\|_{\wedge} \leq \operatorname{dim} Y / \operatorname{dim} X$. It follows easily that if $X$ is an infinitedimensional Banach space and $\mathcal{A}(X)$ has an approximate diagonal $\left(d_{\alpha}\right)_{\alpha \in I}$ such that, for every $\alpha \in I$,

(i) $\pi\left(d_{\alpha}\right)$ is a projection,

(ii) $w d_{\alpha}=d_{\alpha} w\left(w \in \mathcal{F}\left(\operatorname{rg}\left(\pi\left(d_{\alpha}\right)\right)\right)\right)$,

(iii) $w d_{\alpha}^{\circ}=d_{\alpha}^{\circ} w\left(w \in \mathcal{F}\left(\operatorname{rg}\left(\pi\left(d_{\alpha}^{\circ}\right)\right)\right)\right)$,

(iv) $\left\|d_{\alpha}\right\|_{\wedge}=1$, and

(v) $\operatorname{rk}\left(\pi\left(d_{\alpha}^{\circ}\right)\right) \leq C \operatorname{rk}\left(\pi\left(d_{\alpha}\right)\right)$ for some constant $C$ independent of $\alpha$,

then $\mathcal{A}(X)$ has property $\mathbb{A}$ with constant $\leq C$. Note that the first and second assumptions on $\left(d_{\alpha}\right)$ are not significant restrictions. Indeed, as indicated in Section 2 , if $X$ is a $\pi$-space then $\left(d_{\alpha}\right)$ can always be chosen to satisfy both of them. 
6. On the existence of a Banach space $X$ without property $\mathbb{A}$ and with $\mathcal{A}(X)$ amenable. In [GJW, Theorem 6.5], it was shown that every Banach space of the form $\left(\bigoplus_{k=1}^{\infty} \ell_{p}^{n_{k}}\right)_{\ell_{q}}$, where $1 \leq p \neq q \leq \infty$ and $\lim \sup _{k} n_{k}=\infty$, carries an amenable algebra of approximable operators. However, the question of whether or not these spaces have property $\mathbb{A}$ was left open in that paper. For this reason, it was expected that Banach spaces of this form could provide an affirmative answer to the problem in the title of this section. Let us start by showing that this is not the case. In fact, quite the opposite, every Banach space of this form has property $\mathbb{A}$.

The idea of the proof is simple. For instance, if $n_{k}=k(k \in \mathbb{N})$ then, for every $n \in \mathbb{N}$,

$$
\left(\bigoplus_{k=1}^{2 n} \ell_{p}^{k}\right)_{\ell_{q}} \cong\left(\bigoplus_{k=1}^{n}\left(\ell_{p}^{k} \oplus_{q} \ell_{p}^{2 n-k+1}\right)\right)_{\ell_{q}}
$$

and

$$
d\left(\left(\bigoplus_{k=1}^{n}\left(\ell_{p}^{k} \oplus_{q} \ell_{p}^{2 n-k+1}\right)\right)_{\ell_{q}},\left(\bigoplus_{k=1}^{n} \ell_{p}^{2 n+1}\right)_{\ell_{q}}\right) \leq 2^{(q-1) / q} .
$$

Since the symmetric diagonal for $\mathcal{A}\left(\left(\bigoplus_{k=1}^{n} \ell_{p}^{2 n+1}\right)_{\ell_{q}}\right)$ has norm 1 (see BG2, Proposition 3.7]), the symmetric diagonal for $\mathcal{A}\left(\left(\bigoplus_{k=1}^{n}\left(\ell_{p}^{k} \oplus_{q} \ell_{p}^{2 n-k+1}\right)\right)_{\ell_{q}}\right)$ should have norm $\leq 2^{2(q-1) / q}$, and so $\left(\bigoplus_{k=1}^{\infty} \ell_{p}^{k}\right)_{\ell_{q}}$ has property $\mathbb{A}$ with constant $\leq 2^{2(q-1) / q}$.

We shall prove here a result slightly stronger than the one mentioned above. For this, we shall rely on the following particular case of JRZ, Lemma 2.4].

Lemma 6.1. Let $X$ be a Banach space and let $P: X \rightarrow E$ be a finiterank projection onto the subspace $E$ of $X$. If $F$ is a linear subspace of $X$ such that $\operatorname{dim} F=\operatorname{dim} E$ and $\left\|\left.(P-\mathrm{id})\right|_{F}\right\| \leq \varepsilon$, where $\varepsilon \in(0,1)$ satisfies $(1-\varepsilon)^{-1} \varepsilon \operatorname{dim} E<1$, then there is a projection $U: X \rightarrow F$ such that

$$
\|U-P\| \leq(1-\varepsilon)^{-1} \varepsilon \operatorname{dim} E\|P\| .
$$

Recall that, given an infinite-dimensional Banach space $E$ with a 1unconditional Schauder basis $\mathbf{e}=\left(e_{i}\right)$ and a sequence of Banach spaces $\left(X_{i},\|\cdot\|_{i}\right)$, the Banach space $\left(\bigoplus_{i} X_{i}\right)_{\mathbf{e}}$ is defined to be the linear space $\left\{\left(x_{i}\right) \in \Pi_{i} X_{i}\right.$ : the series $\sum_{i}\left\|x_{i}\right\|_{i} e_{i}$ converges in $\left.E\right\}$, endowed with the norm $\left\|\left(x_{i}\right)\right\|=\left\|\sum_{i}\right\| x_{i}\left\|_{i} e_{i}\right\|_{E}$. If $X_{i}=\{0\}$ for every $i>m$ then we also write $\left(\bigoplus_{i=1}^{m} X_{i}\right)_{\mathbf{e}}$. Furthermore, if for some Banach space $X$ and $m \in \mathbb{N}$, $X_{i}=X$ for every $1 \leq i \leq m$ and $X_{i}=\{0\}$ for every $i>m$ (resp. $X_{i}=X$ for every $i \in \mathbb{N}$ ) then we write $E^{m}(X)$ (resp., $\left.E(X)\right)$ instead of $\left(\bigoplus_{i=1}^{m} X_{i}\right)_{\mathbf{e}}$ (resp. $\left.\left(\bigoplus_{i} X_{i}\right)_{\mathbf{e}}\right)$. 
Proposition 6.2. Let $X$ be a Banach space and let $\left(p_{i}\right)$ be a bounded sequence of projections in $\mathcal{A}(X)$ with a subsequence converging strongly to $\mathrm{id}_{X}$. Let $X_{i}:=\operatorname{rg} p_{i}(i \in \mathbb{N})$ and let $E$ be a Banach space with a 1-unconditional shrinking Schauder basis $\mathbf{e}:=\left(e_{i}\right)$. If $\mathcal{A}(E(X))$ has property $\mathbb{A}$ with constant $M$ then $\mathcal{A}\left(\left(\bigoplus_{i} X_{i}\right)_{\mathbf{e}}\right)$ has property $\mathbb{A}$ with constant

$$
\leq 4 M\left(2 \sup _{i}\left\|p_{i}\right\|+M\right)^{2}\left(\sup _{i}\left\|p_{i}\right\|+M\right)^{2} .
$$

Proof. Let $\left(d_{\alpha}\right)_{\alpha \in J}$ be an approximate diagonal for $\mathcal{A}(E(X))$ of norm $\leq M$ such that, for every $\alpha \in J, d_{\alpha}$ is the symmetric diagonal for $\mathcal{A}\left(\operatorname{rg} Q_{\alpha}\right)$, where $Q_{\alpha}:=\pi\left(d_{\alpha}\right)$. Note that each $Q_{\alpha}$ must be a projection. Also, let $\pi_{i}: E(X) \rightarrow X$ (resp., $\left.\imath_{i}: X \rightarrow E(X)\right)$ stand for the $i$ th coordinate projection (resp., embedding), and let $P_{k}:=\sum_{i=1}^{k} \imath_{i} p_{i} \pi_{i}(k \in \mathbb{N})$. Clearly, to prove the desired result, it will suffice to exhibit a bounded sequence $\left(q_{k}\right)_{k \in \mathbb{N}}$ of projections in $\mathcal{A}\left(\left(\bigoplus_{i} X_{i}\right)_{\mathbf{e}}\right)$ converging strongly to the identity map on $\left(\bigoplus_{i} X_{i}\right)_{\mathbf{e}}$ and such that, for every $k \in \mathbb{N}$, there is an $\alpha(=\alpha(k)) \in J$ such that $d\left(\operatorname{rg} q_{k}, \operatorname{rg} Q_{\alpha}\right) \leq C$ for some constant $C$ independent of $k$.

For each $k \in \mathbb{N}$ we construct $q_{k}$ as follows. First choose $\alpha=\alpha(k) \in J$ such that $\operatorname{rg} P_{k} Q_{\alpha} P_{k}=\operatorname{rg} P_{k}$ and

$$
\left\|\left.\left(P_{k}-\mathrm{id}\right)\right|_{\operatorname{rg} Q_{\alpha} P_{k}}\right\|<1 /\left(\operatorname{rk} P_{k}\right)^{2} .
$$

Then choose positive integers $n_{1}<\cdots<n_{N}$ greater than $k$ such that if $R_{k}=\sum_{i=1}^{N} \imath_{i} p_{n_{i}} \pi_{i}$ then $\operatorname{rg} Q_{\alpha} R_{k} Q_{\alpha}=\operatorname{rg} Q_{\alpha}, \operatorname{rg} P_{k} R_{k} Q_{\alpha} P_{k}=\operatorname{rg} P_{k}$, $\left\|\left.\left(P_{k}-\mathrm{id}\right)\right|_{\operatorname{rg} R_{k} Q_{\alpha} P_{k}}\right\| \leq 1 /\left(\operatorname{rk} P_{k}\right)^{2} \quad$ and $\quad\left\|\left.\left(Q_{\alpha}-\mathrm{id}\right)\right|_{\operatorname{rg} R_{k} Q_{\alpha}}\right\| \leq 1 /\left(\operatorname{rk} Q_{\alpha}\right)^{2}$. (The existence of such integers is guaranteed by the fact that $\left(p_{i}\right)$ contains a subsequence that converges strongly to $\mathrm{id}_{X}$.) By Lemma 6.1. for every $k$ large enough, there are projections $U_{k}: E(X) \rightarrow \operatorname{rg} R_{k} Q_{\alpha} P_{k}$ and $V_{k}$ : $E(X) \rightarrow \operatorname{rg} R_{k} Q_{\alpha}$ such that

$$
\left\|U_{k}-P_{k}\right\| \leq\left(\operatorname{rk} P_{k}-1\right)^{-1}\left\|P_{k}\right\| \quad \text { and } \quad\left\|V_{k}-Q_{\alpha}\right\| \leq\left(\operatorname{rk} Q_{\alpha}-1\right)^{-1}\left\|Q_{\alpha}\right\| .
$$

In addition, it follows from our choice of $\alpha$ and $R_{k}$ above that

$$
d\left(\operatorname{rg} U_{k}, \operatorname{rg} P_{k}\right)=d\left(\operatorname{rg} R_{k} Q_{\alpha} P_{k}, \operatorname{rg} P_{k} R_{k} Q_{\alpha} P_{k}\right) \leq \frac{\mathrm{rk} P_{k}+1}{\operatorname{rk} P_{k}-1},
$$

and

$$
d\left(\operatorname{rg} Q_{\alpha}, \operatorname{rg} V_{k}\right)=d\left(\operatorname{rg} Q_{\alpha} R_{k} Q_{\alpha}, \operatorname{rg} R_{k} Q_{\alpha}\right) \leq \frac{\operatorname{rk} Q_{\alpha}+1}{\operatorname{rk} Q_{\alpha}-1},
$$

where we have used the fact that if $\|T x-x\| \leq \varepsilon\|x\|(x \in F)$ for some $0<$ $\varepsilon<1$, then $\left\|\left.T\right|_{F}\right\| \leq 1+\varepsilon,\left\|\left(\left.T\right|_{F}\right)^{-1}\right\| \leq 1-\varepsilon$ and $d(F, T F) \leq(1+\varepsilon) /(1-\varepsilon)$.

Define $Y_{k}:=\operatorname{rg} R_{k}$ and $\widetilde{Y}_{k}:=\left\{\left(x_{i}\right) \in\left(\bigoplus_{i} X_{i}\right)_{\mathbf{e}}: x_{i}=0\right.$ if $i \neq n_{j}$ $(1 \leq j \leq N)\}$. Clearly, $Y_{k} \cong \widetilde{Y}_{k}$, so $d\left(Y_{k}, \widetilde{Y}_{k}\right)=1$. Let us write $\widetilde{U}_{k}$ and $\widetilde{V}_{k}$ for the projections on $\widetilde{Y}_{k}$ corresponding to $\left.U_{k}\right|_{Y_{k}}$ and $\left.V_{k}\right|_{Y_{k}}$, respectively. Also, 
let us write $Z_{k}$ for $\left(\bigoplus_{i=1}^{k} X_{i}\right)_{\mathbf{e}}$. Note that $\operatorname{rg} P_{k} \cong Z_{k}$, so $d\left(\operatorname{rg} P_{k}, Z_{k}\right)=1$. Moreover, if $R: \operatorname{rg} U_{k} \rightarrow Z_{k}$ and $S: \operatorname{rg}\left(V_{k}-U_{k}\right) \rightarrow \operatorname{rg}\left(\widetilde{V}_{k}-\widetilde{U}_{k}\right)$ are linear isomorphisms such that $\|R\|=1,\left\|R^{-1}\right\|=d\left(\operatorname{rg} U_{k}, Z_{k}\right)$ and $\|S\|=\left\|S^{-1}\right\|=1$ then, for every $x \in \operatorname{rg} U_{k}$ and every $y \in \operatorname{rg}\left(V_{k}-U_{k}\right)$,

$$
\begin{aligned}
\|x+y\| & \leq\left(\left\|R^{-1}\right\|+1\right)\|R x+S y\| \\
& \leq\left(\left\|R^{-1}\right\|+1\right)\left(\left\|R U_{k}\right\|+\left\|V_{k}-U_{k}\right\|\right)\|x+y\| \\
& \leq\left(1+d\left(\operatorname{rg} U_{k}, Z_{k}\right)\right)\left(\left\|U_{k}\right\|+\left\|V_{k}-U_{k}\right\|\right)\|x+y\|,
\end{aligned}
$$

so

$$
\begin{aligned}
d\left(\operatorname{rg} U_{k}+\operatorname{rg}\left(V_{k}-U_{k}\right), Z_{k}+\right. & \left.\operatorname{rg}\left(\widetilde{V}_{k}-\widetilde{U}_{k}\right)\right) \\
& \leq\left(1+d\left(\operatorname{rg} U_{k}, Z_{k}\right)\right)\left(\left\|U_{k}\right\|+\left\|V_{k}-U_{k}\right\|\right) .
\end{aligned}
$$

Thus,

$$
\begin{aligned}
d\left(\operatorname{rg} Q_{\alpha}, Z_{k}\right. & \left.+\operatorname{rg}\left(\widetilde{V}_{k}-\widetilde{U}_{k}\right)\right) \\
& \leq d\left(\operatorname{rg} Q_{\alpha}, \operatorname{rg} V_{k}\right) d\left(\operatorname{rg} U_{k}+\operatorname{rg}\left(V_{k}-U_{k}\right), Z_{k}+\operatorname{rg}\left(\widetilde{V}_{k}-\widetilde{U}_{k}\right)\right) \\
& \leq d\left(\operatorname{rg} Q_{\alpha}, \operatorname{rg} V_{k}\right)\left(1+d\left(\operatorname{rg} U_{k}, Z_{k}\right)\right)\left(\left\|U_{k}\right\|+\left\|V_{k}-U_{k}\right\|\right),
\end{aligned}
$$

and so, given an arbitrary $\varepsilon>0$, we should have, for $k$ large enough,

$$
d\left(\operatorname{rg} Q_{\alpha}, Z_{k}+\operatorname{rg}\left(\widetilde{V}_{k}-\widetilde{U}_{k}\right)\right) \leq(2+\varepsilon)\left(\left\|P_{k}\right\|+\left\|Q_{\alpha}-P_{k}\right\|\right) .
$$

We then define $q_{k}$ to be the composite of the natural projection from $\left(\bigoplus_{i} X_{i}\right)_{\mathbf{e}}$ onto $Z_{k}+\widetilde{Y}_{k}$ with the projection $\operatorname{id}_{Z_{k}} \oplus\left(\widetilde{V}_{k}-\widetilde{U}_{k}\right): Z_{k}+\widetilde{Y}_{k} \rightarrow$ $Z_{k}+\operatorname{rg}\left(\widetilde{V}_{k}-\widetilde{U}_{k}\right)$. One easily sees that the sequence $\left(q_{k}\right)$ satisfies all required conditions.

As for the property $\mathbb{A}$ constant of $\mathcal{A}\left(\left(\bigoplus_{i} X_{i}\right)_{\mathbf{e}}\right)$, simply note that if $\sum_{i} R_{i, \alpha} \otimes S_{i, \alpha} \in \mathcal{F}\left(\operatorname{rg} Q_{\alpha}\right) \otimes \mathcal{F}\left(\operatorname{rg} Q_{\alpha}\right)$ is a representation of $d_{\alpha}(\alpha \in J)$ and $T_{k}: \operatorname{rg} Q_{\alpha(k)} \rightarrow \operatorname{rg} q_{k}$ is a linear isomorphism $(k \in \mathbb{N})$ then $\sum_{i} T_{k} R_{i, \alpha(k)} T_{k}^{-1} q_{k}$ $\otimes T_{k} S_{i, \alpha(k)} T_{k}^{-1} q_{k}$ is the symmetric diagonal for $\mathcal{A}\left(\operatorname{rg} q_{k}\right)$. Thus, the property A constant of $\mathcal{A}\left(\left(\bigoplus_{i} X_{i}\right)_{\mathbf{e}}\right)$ cannot exceed

$M \liminf _{k} d\left(\operatorname{rg} q_{k}, \operatorname{rg} Q_{\alpha}\right)^{2}\left\|q_{k}\right\|^{2} \leq 4 M\left(2 \sup _{i}\left\|p_{i}\right\|+M\right)^{2}\left(\sup _{i}\left\|p_{i}\right\|+M\right)^{2}$.

Although the estimates provided by Proposition 6.2 are far from sharp, we suspect that, in general, if $E$ and $X$ are incomparable Banach spaces then the property $\mathbb{A}$ constant of $\left(\bigoplus_{k}\left[x_{i}\right]_{i=1}^{n_{k}}\right)_{\mathbf{e}}$ will be greater than the property $\mathbb{A}$ constants of both $E$ and $X$. We do not have a proof for this, not even in the case where both $E$ and $X$ are $\ell_{p}$-spaces. However, assuming this were true for $\ell_{p}$-spaces, and bearing in mind that $\mathcal{A}\left(\left(\bigoplus_{k} \ell_{p}^{n_{k}}\right) \ell_{q}\right)$ is 1-amenable whenever $\limsup _{k} n_{k}=\infty([\mathrm{BG} 2$, Example 3.6]), one might wonder whether iterating this construction and taking some kind of direct limit one could produce a Banach space $X$ carrying an amenable algebra of approximable operators 
and yet lacking property $\mathbb{A}$. Our next result shows why this idea is unlikely to work.

Corollary 6.3. Let $1 \leq p_{1}, \ldots, p_{m+1} \leq \infty$, and let $\left(n_{i, 1}\right),\left(n_{i, 2}\right), \ldots$, $\left(n_{i, m}\right)$ be sequences of non-negative integers. Define $E_{1}:=\ell_{p_{1}}$ and $E_{k+1}:=$ $\left(\bigoplus_{i} \ell_{p_{k+1}}^{n_{i, k}}\right)_{\mathbf{e}(k)}(1 \leq k \leq m)$, where $\mathbf{e}(k)$ is the natural unit vector basis of $E_{k}$. If $\lim \sup _{i} n_{i, m}=\sup _{i} n_{i, m}$ then $E_{m+1}$ has property $\mathbb{A}$ with constant $\leq 144$.

Proof. Define $E:=\ell_{p_{1}}\left(\ell_{p_{2}}\left(\cdots\left(\ell_{p_{m}}\right) \cdots\right)\right)$. It is known that $\mathcal{A}(E)$ has property $\mathbb{A}$ with constant 1 . (This follows easily by induction combined with an argument very similar to that used in the proof of [BG2, Proposition 3.7].) It is clear that the unit vector basis of $E$ can be reordered so as to contain the unit vector basis of $E_{k}$ as a subsequence. Denote by e the new (reordered) basis for $E$. Then one can realize $E_{m+1}$ as a space of the form $\left(\bigoplus_{i} \ell_{p_{m+1}}^{n_{i}}\right)_{\mathbf{e}}$ for some sequence $\left(n_{i}\right)$ of non-negative integers. Of course, one may have $n_{i}=0$ for infinitely many values of $i$. Now, let $\gamma:=\sup _{i} n_{i, m}$ (if $\left(n_{i, m}\right)$ is unbounded then let $\gamma=\infty)$. By hypothesis, $\left(n_{i, m}\right)$ contains a subsequence converging to $\gamma$, and since $\left(n_{i}\right)$ contains $\left(n_{i, m}\right)$ as a subsequence, also $\left(n_{i}\right)$ has a subsequence converging to $\gamma$. In turn, this means that $\left(q_{i}\right) \subset \mathcal{A}\left(\ell_{p_{m+1}}^{\gamma}\right)$, where $q_{i}$ stands for the natural projection onto the linear span of the first $n_{i}$ vectors of the unit vector basis of $\ell_{p_{m+1}}^{\gamma}$, has a subsequence converging strongly to the identity map on $\ell_{p_{m+1}}^{\gamma}$ (if $\gamma=\infty$ then $\ell_{p_{m+1}}^{\gamma}=\ell_{p_{m+1}}$ ). Thus, by Proposition 6.2, $E_{m+1}$ has property $\mathbb{A}$ with constant $\leq 4(2+1)^{2}(1+1)^{2}$. (Actually, in this case, it is not hard to see, from the proof of Proposition 6.2. that the property $\mathbb{A}$ constant of $E_{m+1}$ should be $\leq 4$.)

REMARK 6.4. Note that starting with numbers $1 \leq p_{1}, \ldots, p_{m+1} \leq \infty$ and sequences $\left(n_{k, 1}\right),\left(n_{k, 2}\right), \ldots,\left(n_{k, m}\right)$ as in the statement of Corollary 6.3 one could, alternatively, define $F_{1}:=\ell_{p_{1}}$ and then $F_{k+1}:=\left(\bigoplus_{i}\left[e_{j, k}\right]_{j=1}^{n_{i, k}}\right)_{\ell_{p_{k+1}}}$ $(1 \leq k \leq m)$, where $\left(e_{j, k}\right)_{j \in \mathbb{N}}$ is the natural unit vector basis for $F_{k}$. However, it is not hard to see that if one drops the assumption on $\left(n_{i, m}\right)$ then this construction will produce exactly the same family of Banach spaces as the construction considered in Corollary 6.3.

In view of the above results, one is naturally led to ask whether there are other families of Banach spaces that could yield the desired example. Our next result will provide a class of Banach spaces, essentially different from the one considered thus far, all of whose members lack property $\mathbb{A}$. We think it could contain examples of Banach spaces $X$ for which $\mathcal{A}(X)$ is amenable, although, so far, we have failed to find any. Recall first that an operator ideal norm on the operator ideal $\mathcal{F}$ of all finite-rank operators between Banach spaces is a function $\gamma: \mathcal{F} \rightarrow[0, \infty[$ that satisfies the following:

(a) $\left.\gamma\right|_{\mathcal{F}(X, Y)}$ is a norm for every pair of Banach spaces $X$ and $Y$;

(b) $\gamma\left(\right.$ id $\left._{\mathbb{C}}: \mathbb{C} \rightarrow \mathbb{C}\right)=1$; and 
(c) if $A: Y \rightarrow Y_{0}$ and $B: X_{0} \rightarrow X$ are bounded operators and $T \in$ $\mathcal{F}(X, Y)$ then $\gamma(A T B) \leq\|A\| \gamma(T)\|B\|$.

Proposition 6.5. Let $X$ be a Banach space. Suppose that for some $\delta>0$ and some operator ideal norm $\gamma$ on $\mathcal{F}$ there is a map $P \mapsto Q_{P}$ from the set of all projections in $\mathcal{A}(X)$ into itself such that, for every projection $P \in \mathcal{A}(X), P Q_{P}=Q_{P}=Q_{P} P$ and $\operatorname{rk} Q_{P} \geq \delta \mathrm{rk} P$; and, whenever $\left(P_{\alpha}\right) \subset \mathcal{A}(X)$ is a bounded net of projections converging strongly to $\mathrm{id}_{X}$, $\lim \sup _{\alpha} \gamma\left(P_{\alpha}\right) / \gamma\left(Q_{P_{\alpha}}\right)=\infty$. Then $\mathcal{A}(X)$ cannot have a bounded approximate diagonal $\left(d_{\alpha}\right)_{\alpha \in I}$ such that, for every $\alpha \in I$,

(i) $P_{\alpha}:=\pi\left(d_{\alpha}\right)$ is a projection;

(ii) $\pi\left(d_{\alpha}^{\circ}\right)=\pi\left(d_{\alpha}\right)$; and

(iii) $P_{\alpha} d_{\alpha}=d_{\alpha}$ and $W d_{\alpha}=d_{\alpha} W\left(W \in P_{\alpha} \mathcal{A}(X) P_{\alpha}\right)$.

Proof. Suppose towards a contradiction that $\mathcal{A}(X)$ has a bounded approximate diagonal $\left(d_{\alpha}\right)_{\alpha \in I}$ satisfying conditions (i)-(iii) above.

For each $\alpha \in I$, let $\sum_{i} R_{i, \alpha} \otimes S_{i, \alpha}$ be a representation of $d_{\alpha}$ such that $\sum_{i}\left\|R_{i, \alpha}\right\|\left\|S_{i, \alpha}\right\| \leq 2\left\|d_{\alpha}\right\|_{\wedge}$, and let $Q_{\alpha}=Q_{P_{\alpha}}$. Condition (iii) implies that $\pi\left(\sum_{i} R_{i, \alpha} \otimes Q_{\alpha} S_{i, \alpha}\right)=c P_{\alpha}$ for some scalar $c$. Moreover, $c \geq \delta$, for

$$
\begin{aligned}
c\left(\operatorname{rk} P_{\alpha}\right) & =\operatorname{tr}\left(c P_{\alpha}\right)=\operatorname{tr}\left(\pi\left(\sum_{i} R_{i, \alpha} \otimes Q_{\alpha} S_{i, \alpha}\right)\right) \\
& =\operatorname{tr}\left(\sum_{i} R_{i, \alpha} Q_{\alpha} S_{i, \alpha}\right)=\operatorname{tr}\left(\left(\sum_{i} S_{i, \alpha} R_{i, \alpha}\right) Q_{\alpha}\right)=\operatorname{rk} Q_{\alpha}
\end{aligned}
$$

(where we have used (i) and (ii)). Then note that

$$
\gamma\left(c P_{\alpha}\right) \leq \sum_{i} \gamma\left(R_{i, \alpha} Q_{\alpha} S_{i, \alpha}\right) \leq \sum_{i}\left\|R_{i, \alpha}\right\| \gamma\left(Q_{\alpha}\right)\left\|S_{i, \alpha}\right\| \leq 2\left\|d_{\alpha}\right\|_{\wedge} \gamma\left(Q_{\alpha}\right),
$$

and hence

$$
\gamma\left(P_{\alpha}\right) / \gamma\left(Q_{\alpha}\right) \leq 2\left\|d_{\alpha}\right\|_{\wedge} / \delta \quad(\alpha \in I),
$$

which contradicts our hypothesis.

The conditions of Proposition 6.5 have been modeled on the defining properties of weak Hilbert spaces. Recall that weak Hilbert spaces are Banach spaces which are both weak type and weak cotype 2. A well known characterization of them states that a weak Hilbert space is a Banach space $X$ such that for every $0<\delta<1$, there is a constant $C(\delta)$ with the following property: every finite-dimensional subspace $E \subset X$ contains a subspace $F \subset E$ with $\operatorname{dim} F \geq \delta \operatorname{dim} E$ such that $d\left(F, \ell_{2}^{\operatorname{dim} F}\right) \leq C(\delta)$ and there is a projection $P: X \rightarrow F$ with $\|P\| \leq C(\delta)$ ( rem 12.2]). So, in particular, every weak Hilbert space non-isomorphic to a Hilbert space satisfies the conditions of Proposition 6.5 with $\gamma=\gamma_{2}$ (recall $\gamma_{2}(T: X \rightarrow Y)=\inf \left\{\|R\|\|S\|: R: \ell_{2} \rightarrow Y, S: X \rightarrow \ell_{2}\right.$ and $\left.\left.R S=T\right\}\right)$. The class of weak Hilbert spaces ranges from the classical Hilbert spaces, 
all of which have property $\mathbb{A}$ with constant 1 , to the 2 -convexified Tsirelson spaces, which fail to be amenable. These spaces have been extensively studied. However, if one restricts attention to this class then the problem gets slightly more difficult, as indicated by our next result, which may be seen as an extension of [CS, Theorem Ae15].

Proposition 6.6. Let $H$ be a weak Hilbert space. If $\mathcal{A}(H)$ has a bounded approximate diagonal $\left(d_{\alpha}\right)_{\alpha \in I}$ such that, for every $\alpha \in I$,

(i) $P_{\alpha}:=\pi\left(d_{\alpha}\right)$ is a projection;

(ii) $\pi\left(d_{\alpha}^{\circ}\right)$ is a scalar multiple of a projection; and

(iii) $P_{\alpha} d_{\alpha}=d_{\alpha}$ and $W d_{\alpha}=d_{\alpha} W\left(W \in P_{\alpha} \mathcal{A}(X) P_{\alpha}\right)$,

then $H$ is isomorphic to a Hilbert space.

Proof. Suppose towards a contradiction that $\mathcal{A}(H)$ has a bounded approximate diagonal $\left(d_{\alpha}\right)_{\alpha \in I}$ with the indicated properties. Set $H_{\alpha}=\operatorname{rg} P_{\alpha}$ $(\alpha \in I)$. Recall that, since $H$ is a weak Hilbert space, there are constants $0<\delta \leq 1$ and $C \geq 1$ such that for every finite-dimensional subspace $X$ of $H$ there exist a linear subspace $Y \subseteq X$ with $\operatorname{dim} Y \geq \delta \operatorname{dim} X$ and $d\left(Y, \ell_{2}^{\operatorname{dim} Y}\right) \leq C$, and a projection $Q: H \rightarrow Y$ with $\|Q\| \leq C$.

Let $d_{\alpha}=\sum_{i=1}^{m(\alpha)} R_{i}^{\alpha} \otimes S_{i}^{\alpha}$, where the $R_{i}^{\alpha}$ 's and the $S_{i}^{\alpha}$ 's have been chosen in such a way that $\sum_{i}\left\|R_{i}^{\alpha}\right\|^{2}=1$ and $\left(\sum_{i}\left\|S_{i}^{\alpha}\right\|^{2}\right)^{1 / 2}=\left\|d_{\alpha}\right\|_{\wedge}$, and let $H_{\alpha}^{\circ}=$ $\operatorname{rg} \pi\left(d_{\alpha}^{\circ}\right)$. By the previous paragraph, there exist a subspace $K_{\alpha}$ of $H_{\alpha}^{\circ}$ and a projection $Q_{\alpha}: H \rightarrow K_{\alpha}$ such that $\operatorname{dim} K_{\alpha} \geq \delta \operatorname{dim} H_{\alpha}^{\circ}, d\left(K_{\alpha}, \ell_{2}^{\operatorname{dim} K_{\alpha}^{\alpha}}\right) \leq C$ and $\left\|Q_{\alpha}\right\| \leq C$. Define $\widetilde{d}_{\alpha}:=\sum_{i} R_{i}^{\alpha} \jmath_{\alpha} \otimes Q_{\alpha} S_{i}^{\alpha}$, where we have written $\jmath_{\alpha}$ for the natural inclusion of $K_{\alpha}$ into $H$. For each $i$, let $X_{i}^{\alpha} \cong \ell_{2}^{\operatorname{dim} K_{\alpha}}$ and let $T_{i}$ : $K_{\alpha} \rightarrow X_{i}^{\alpha}$ be a norm-1 linear isomorphism such that $\left\|T_{i}^{-1}\right\|=d\left(K_{\alpha}, X_{i}^{\alpha}\right)$. Define $X_{\alpha}:=\left(\bigoplus_{i} X_{i}^{\alpha}\right)_{\ell_{2}}$, and let $\pi_{i}: X_{\alpha} \rightarrow X_{i}^{\alpha}$ and $\imath_{i}: X_{i}^{\alpha} \rightarrow X_{\alpha}$ be the $i$ th coordinate projection and embedding, respectively. Lastly, define $R:=\sum_{i} R_{i}^{\alpha} \jmath_{\alpha} T_{i}^{-1} \pi_{i}$ and $S:=\sum_{i} \imath_{i} T_{i} Q_{\alpha} S_{i}^{\alpha}$. Clearly, $R S=\pi\left(\widetilde{d}_{\alpha}\right)=\varkappa P_{\alpha}$ for some scalar $\varkappa$. Let $\operatorname{tr}\left(\pi\left(d_{\alpha}^{\circ}\right)\right)=c \operatorname{dim} H_{\alpha}^{\circ}$ and note that $\operatorname{tr}\left(\pi\left(d_{\alpha}^{\circ}\right)\right)=$ $\operatorname{tr}\left(\pi\left(d_{\alpha}\right)\right)=\operatorname{dim} H_{\alpha}$, so

$$
\begin{aligned}
\varkappa \operatorname{dim} H_{\alpha} & =\operatorname{tr}\left(\pi\left(\widetilde{d}_{\alpha}\right)\right)=\operatorname{tr}\left(\pi\left(\widetilde{d}_{\alpha}^{\circ}\right)\right) \\
& =\operatorname{tr}\left(Q_{\alpha} \pi\left(d_{\alpha}^{\circ}\right) \jmath\right)=\frac{\operatorname{dim} H_{\alpha}}{\operatorname{dim} H_{\alpha}^{\circ}} \operatorname{tr}\left(\operatorname{id}_{K_{\alpha}}\right) \geq \delta \operatorname{dim} H_{\alpha},
\end{aligned}
$$

i.e., $\varkappa \geq \delta$. Moreover, for every $\left(x_{1}, \ldots, x_{m}\right) \in X_{\alpha}$,

$$
\begin{aligned}
\left\|R\left(x_{1}, \ldots, x_{m}\right)\right\| & =\left\|\sum_{i} R_{i}^{\alpha} \jmath_{\alpha} T_{i}^{-1}\left(x_{i}\right)\right\| \\
& \leq\left(\sum_{i}\left\|R_{i}^{\alpha} \jmath_{\alpha} T_{i}^{-1}\right\|^{2}\right)^{1 / 2}\left(\sum_{i}\left\|x_{i}\right\|^{2}\right)^{1 / 2} \\
& \leq C\left\|\left(x_{1}, \ldots, x_{m}\right)\right\|,
\end{aligned}
$$


and, for every $h \in H_{\alpha}$,

$$
\|S(h)\|=\left(\sum_{i}\left\|T_{i} Q_{\alpha} S_{i}^{\alpha}(h)\right\|^{2}\right)^{1 / 2} \leq C\left\|d_{\alpha}\right\|_{\wedge}\|h\| .
$$

Thus, $d\left(H_{\alpha}, \ell_{2}^{\operatorname{dim} H_{\alpha}}\right)=d\left(H_{\alpha}, \operatorname{rg} R\right) \leq \varkappa^{-1}\|R\|\|S\| \leq \delta^{-1} C^{2}\left\|d_{\alpha}\right\|_{\wedge}$.

Since the net $\left(P_{\alpha}\right)$ converges strongly to id ${ }_{H}$, it follows easily from our last estimate that $d\left(E, \ell_{2}^{\operatorname{dim} E}\right) \leq \delta^{-1} C^{2} \sup _{\alpha}\left\|d_{\alpha}\right\|_{\wedge}$ for every finite-dimensional subspace $E$ of $H$. In turn, by [LP, Proposition 5.2], we conclude that $H$ is isomorphic to a Hilbert space.

We finish the note with the following question:

Is it possible for a weak Hilbert space which is not isomorphic to a Hilbert space to carry an amenable Banach algebra of approximable operators?

In view of the above, an affirmative answer to this question would imply a positive answer to the problem in the title of the section.

\section{References}

[BD] F. F. Bonsall and J. Duncan, Numerical Ranges. II, London Math. Soc. Lecture Note Ser. 10, Cambridge Univ. Press, New York, 1973.

[BG1] A. Blanco and N. Grønbæk, Cohomology of Banach algebras of operators and geometry of Banach spaces, in: Function Spaces, Contemp. Math. 435, Amer. Math. Soc., Providence, RI, 2007, 57-80.

[BG2] A. Blanco and N. Grønbæk, Amenability of algebras of approximable operators, Israel J. Math. 171 (2009), 127-156.

[BK] H. F. Bohnenblust and S. Karlin, Geometrical properties of the unit sphere of Banach algebras, Ann. of Math. (2) 62 (1955), 217-229.

[CS] P. G. Casazza and T. J. Shura, Tsirelson's Space, Lecture Notes in Math. 1363, Springer, Berlin, 1989.

[GJW] N. Grønbæk, B. E. Johnson and G. A. Willis, Amenability of Banach algebras of compact operators, Israel J. Math. 87 (1994), 289-324.

[Gr] R. Grzaślewicz, Exposed points of the unit ball of $\mathcal{L}(H)$, Math. Z. 193 (1986), 595-596.

[GS] R. Grząślewicz and P. Scherwentke, On strongly extreme and denting points in $\mathcal{L}(H)$, Math. Japon. 41 (1995), 283-284.

[J1] B. E. Johnson, Cohomology in Banach algebras, Mem. Amer. Math. Soc. 127 (1972).

[J2] B. E. Johnson, Approximate diagonals and cohomology of certain annihilator Banach algebras, Amer. J. Math. 94 (1972), 685-698.

[J3] B. E. Johnson, Symmetric amenability and the nonexistence of Lie and Jordan derivations, Math. Proc. Cambridge Philos. Soc. 120 (1996), 455-473.

[JRZ] W. B. Johnson, H. P. Rosenthal and M. Zippin, On bases, finite dimensional decompositions and weaker structures in Banach spaces, Israel J. Math. 9 (1971), $488-506$.

[KW] N. J. Kalton and G. V. Wood, Orthonormal systems in Banach spaces and their applications, Math. Proc. Cambridge Philos. Soc. 79 (1976), 493-510. 
[LP] J. Lindenstrauss and A. Pełczyński, Absolutely summing operators in $\mathcal{L}_{p}$-spaces and their applications, Studia Math. 29 (1968), 275-326.

[LT] J. Lindenstrauss and L. Tzafriri, Classical Banach Spaces I, Sequence Spaces, Ergeb. Math. Grenzgeb. 92, Springer, Berlin, 1977.

[Lu] G. Lumer, Semi-inner-product spaces, Trans. Amer. Math. Soc. 100 (1961), 2943.

[Pi1] G. Pisier, Factorization of Linear Operators and Geometry of Banach Spaces, CBMS Reg. Conf. Ser. Math. 60, Amer. Math. Soc., Providence, RI, 1986.

[Pi2] G. Pisier, The Volume of Convex Bodies and Banach Space Geometry, Cambridge Tracts in Math. 94, Cambridge Univ. Press, Cambridge, 1989.

[Ra1] B. Randrianantoanina, 1-complemented subspaces of spaces with 1-unconditional bases, Canad. J. Math. 49 (1997), 1242-1264.

[Ra2] B. Randrianantoanina, Norm-one projections in Banach spaces, Taiwanese J. Math. 5 (2001), 35-95.

[ST] H. Schneider and R. E. L. Turner, Matrices Hermitian for an absolute norm, Linear Multilinear Algebra 1 (1973), 9-31.

[TJ] N. Tomczak-Jaegermann, Banach-Mazur Distances and Finite-Dimensional Operator Ideals, Pitman Monogr. Surveys Pure Appl. Math. 38, Longman, 1989.
A. Blanco
Department of Pure Mathematics
Queen's University Belfast
Belfast BT7 1NN, UK
E-mail: a.blanco@qub.ac.uk

Received March 1, 2011

Revised version December 25, 2012 